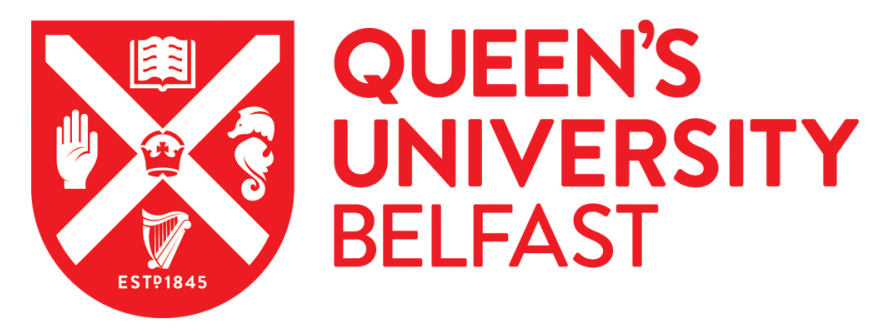

\title{
The Kepler-454 system: a small, not-rocky inner planet, a Jovian world, and a distant companion
}

Gettel, S., Charbonneau, D., Dressing, C. D., Buchhave, L. A., Dumusque, X., Vanderburg, A., Bonomo, A. S., Malavolta, L., Pepe, F., Cameron, A. C., Latham, D. W., Udry, S., Marcy, G. W., Isaacson, H., Howard, A. W., Davies, G. R., Aguirre, V. S., Kjeldsen, H., Bedding, T. R., ... Chaplin, W. J. (2016). The Kepler-454 system: a small, not-rocky inner planet, a Jovian world, and a distant companion. Astrophysical Journal, 816(2), [95]. https://doi.org/10.3847/0004-637X/816/2/95

Published in:

Astrophysical Journal

Document Version:

Publisher's PDF, also known as Version of record

Queen's University Belfast - Research Portal:

Link to publication record in Queen's University Belfast Research Portal

Publisher rights

(C) 2016. The American Astronomical Society. All rights reserved.

General rights

Copyright for the publications made accessible via the Queen's University Belfast Research Portal is retained by the author(s) and / or other copyright owners and it is a condition of accessing these publications that users recognise and abide by the legal requirements associated with these rights.

Take down policy

The Research Portal is Queen's institutional repository that provides access to Queen's research output. Every effort has been made to ensure that content in the Research Portal does not infringe any person's rights, or applicable UK laws. If you discover content in the Research Portal that you believe breaches copyright or violates any law, please contact openaccess@qub.ac.uk. 

COMPANION

Sara Gettel $^{1}$, David Charbonneau ${ }^{1}$, Courtney D. Dressing ${ }^{1,2,3}$, Lars A. Buchhave ${ }^{1,4}$, Xavier Dumusque $^{1}$, Andrew Vanderburg ${ }^{1}$, Aldo S. Bonomo ${ }^{5}$, Luca Malavolta ${ }^{6,7}$, Francesco Pepe $^{8}$, Andrew Collier Cameron ${ }^{9}$, David W. Latham ${ }^{1}$, Stéphane Udry ${ }^{8}$, Geoffrey W. Marcy ${ }^{10}$, Howard IsaAcson ${ }^{10}$, Andrew W. Howard ${ }^{11}$, Guy R. Davies ${ }^{12,13}$, Victor Silva Aguirre ${ }^{13}$, Hans Kueldsen ${ }^{13}$, Timothy R. Bedding ${ }^{13,14}$, Eric Lopez ${ }^{15}$, Laura Affer ${ }^{16}$, Rosario Cosentino ${ }^{17}$, Pedro Figueira ${ }^{18}$, Aldo F. M. Fiorenzano ${ }^{17}$, Avet Harutyunyan ${ }^{17}$, John Asher Johnson ${ }^{1}$, Mercedes Lopez-Morales ${ }^{1}$, Christophe Lovis ${ }^{8}$, Michel Mayor ${ }^{8}$, Giusi Micela ${ }^{16}$, Emilio Molinari ${ }^{17,19}$, Fatemeh Motalebi $^{8}$, David F. Phillips ${ }^{1}$, Giampaolo Piotto ${ }^{6,7}$, Didier Queloz ${ }^{8,20}$, Ken Rice ${ }^{15}$, Dimitar Sasselov ${ }^{1}$, Damien Ségransan ${ }^{8}$, Alessandro Sozzetti ${ }^{5}$, Chris Watson ${ }^{21}$, Sarbani Basu ${ }^{22}$, Tiago L. Campante ${ }^{12,13}$, Jørgen Christensen-Dalsgaard ${ }^{13}$, Steven D. Kawaler ${ }^{23}$, Travis S. Metcalfe ${ }^{24}$, Rasmus Handberg ${ }^{13}$, Mikkel N. Lund ${ }^{13}$, Mia S. LundKvist ${ }^{13}$, Daniel Huber ${ }^{13,14}$, and William J. Chaplin ${ }^{12,13}$

${ }^{1}$ Harvard-Smithsonian Center for Astrophysics, 60 Garden Street, Cambridge, MA 02138, USA; sara.gettel@gmail.com ${ }^{2}$ California Institute of Technology, 1200 E California Boulevard, Pasadena, CA 9112 ${ }^{3}$ NASA Sagan Fellow

${ }^{4}$ Centre for Star and Planet Formation, Natural History Museum of Denmark, University of Copenhagen, DK-1350 Copenhagen, Denmark 5 INAF-Osservatorio Astrofisico di Torino, via Osservatorio 20, I-10025 Pino Torinese, Italy
${ }^{6}$ Dipartimento di Fisica e Astronomia "Galileo Galilei," Universita'di Padova, Vicolo dell'Osservatorio 3, I-35122 Padova, Italy INAF-Osservatorio Astronomico di Padova, Vicolo dell'Osservatorio 5, I-35122 Padova, Italy ${ }^{8}$ Observatoire Astronomique de l'Université de Genève, $51 \mathrm{ch}$. des Maillettes, 1290 Versoix, Switzerland

${ }^{9}$ SUPA, School of Physics \& Astronomy, University of St. Andrews, North Haugh, St. Andrews Fife, KY16 9SS, UK

${ }^{10}$ University of California, Berkeley, CA, 94720, USA

${ }^{11}$ Institute for Astronomy, University of Hawaii at Manoa, 2680 Woodlawn Drive, Honolulu, HI 96822, USA

${ }^{12}$ School of Physics and Astronomy, University of Birmingham, Edgbaston, Birmingham, B15 2TT, UK

${ }^{13}$ Stellar Astrophysics Centre (SAC), Department of Physics and Astronomy, Aarhus University, Ny Munkegade 120, DK-8000 Aarhus C, Denmark ${ }^{14}$ Sydney Institute for Astronomy, School of Physics, University of Sydney 2006, Australia

${ }^{15}$ SUPA, Institute for Astronomy, University of Edinburgh, Royal Observatory, Blackford Hill, Edinburgh, EH93HJ, UK

${ }_{16}$ INAF-Osservatorio Astronomico di Palermo, Piazza del Parlamento 1, I-90134 Palermo, Italy

${ }^{17}$ INAF-Fundación Galileo Galilei, Rambla José Ana Fernandez Pérez 7, E-38712 Breña Baja, Spain

${ }^{18}$ Instituto de Astrofisica e Ciências do Espaço, Universidade do Porto, CAUP, Rua das Estrelas, PT4150-762 Porto, Portugal

${ }^{19}$ INAF-IASF Milano, via Bassini 15, I-20133 Milano, Italy
${ }^{19}$ Cavendish Laboratory, J J Thomson Avenue, Cambridge CB3 OHE, UK

${ }^{21}$ Astrophysics Research Centre, School of Mathematics and Physics, Queens University, Belfast, UK

${ }^{22}$ Department of Astronomy, Yale University, New Haven, CT, 06520, USA

${ }^{23}$ Department of Physics and Astronomy, Iowa State University, Ames, IA 50011, USA

${ }^{24}$ Space Science Institute, 4750 Walnut Street Suite 205, Boulder, CO 80301, USA

Received 2015 May 29; accepted 2015 November 23; published 2016 January 14

\section{ABSTRACT}

Kepler-454 (KOI-273) is a relatively bright ( $V=11.69 \mathrm{mag})$, Sun-like star that hosts a transiting planet candidate in a 10.6 day orbit. From spectroscopy, we estimate the stellar temperature to be $5687 \pm 50 \mathrm{~K}$, its metallicity to be $[\mathrm{m} / \mathrm{H}]=0.32 \pm 0.08$, and the projected rotational velocity to be $v \sin i<2.4 \mathrm{~km} \mathrm{~s}^{-1}$. We combine these values with a study of the asteroseismic frequencies from short cadence Kepler data to estimate the stellar mass to be $1.028_{-0.03}^{+0.04} M_{\odot}$, the radius to be $1.066 \pm 0.012 R_{\odot}$, and the age to be $5.25_{-1.39}^{+1.41} \mathrm{Gyr}$. We estimate the radius of the 10.6 day planet as $2.37 \pm 0.13 R_{\oplus}$. Using 63 radial velocity observations obtained with the HARPS-N spectrograph on the Telescopio Nazionale Galileo and 36 observations made with the HIRES spectrograph at the Keck Observatory, we measure the mass of this planet to be $6.8 \pm 1.4 M_{\oplus}$. We also detect two additional nontransiting companions, a planet with a minimum mass of $4.46 \pm 0.12 M_{J}$ in a nearly circular 524 day orbit and a massive companion with a period $>10$ years and mass $>12.1 M_{J}$. The 12 exoplanets with radii $<2.7 R_{\oplus}$ and precise mass measurements appear to fall into two populations, with those $<1.6 R_{\oplus}$ following an Earth-like composition curve and larger planets requiring a significant fraction of volatiles. With a density of $2.76 \pm 0.73 \mathrm{~g} \mathrm{~cm}^{-3}$, Kepler-454b lies near the mass transition between these two populations and requires the presence of volatiles and/or $\mathrm{H} / \mathrm{He}$ gas.

Key words: planetary systems - planets and satellites: composition - stars: individual (KOI-273 = KIC 3102384) asteroseismology - techniques: radial velocities

Supporting material: machine-readable tables

\section{INTRODUCTION}

The NASA Kepler mission has detected thousands of planet candidates with radii between 1 and $2.7 R_{\oplus}$ (Borucki et al. 2011; Batalha et al. 2013; Burke et al. 2014; Rowe et al. 2015). The corresponding population of low mass planets was previously detected in radial velocity (RV) surveys (Mayor 
\& Udry 2008; Howard et al. 2009), but notably has no analog in our own solar system. The composition of these objects is not yet well understood; theoretical models predict that some of these intermediate size planets may be predominantly rocky and others may have a large fractional composition of volatiles or a substantial hydrogen envelope (Léger et al. 2004; Valencia et al. 2006; Fortney et al. 2007; Seager et al. 2007; Zeng \& Sasselov 2013). There is presently only a small number of such planets observed to transit and having published mass estimates with a precision better than $20 \%$, while less precise mass estimates are generally not sufficient to distinguish between a rocky compositional model and one that is volatile-rich.

Dressing et al. (2015) raised the intriguing possibility that the small planets with well-measured masses of $<6 M_{\oplus}$, equivalent to about $1.6 R_{\oplus}$, have similar compositions, well approximated by a two-component model with the same $\mathrm{MgSiO}_{3} / \mathrm{Fe}$ ratio as the Earth (Zeng \& Sasselov 2013). Planets larger than $2.0 R_{\oplus}$ are observed to have lower densities, consistent with a significant fraction of volatiles or $\mathrm{H} / \mathrm{He}$ gas and do not follow a single mass-radius relation. Due to the limited number of planets smaller than $2.7 R_{\oplus}$ with precise masses, it is not yet clear how broadly applicable the ironmagnesium silicate model might be.

KOI-273 (KIC 3102384) is a moderately bright solar-like star, with $V=11.69 \mathrm{mag}$ and $K_{\mathrm{p}}=11.46 \mathrm{mag}$. It was observed by Kepler during Quarters 0-17, with short cadence data taken during Quarters 4, 6-12, and 15-17. It was identified by the Kepler pipeline as having a planet candidate in the first four months of long cadence data, with radius $R_{\mathrm{p}}=1.86 R_{\oplus}$ and period $P=10.57$ days. This initial radius measurement of KOI-273.01 falls in between the two classes of planets discussed in Dressing et al. (2015) and so it can provide a test case of the Earth-composition mass-radius relation.

The stellar parameters of KOI-273 were previously determined by a combination of asteroseismology and spectroscopy in Huber et al. (2013). They reported an asteroseismically determined stellar radius $R_{*}=1.081 \pm 0.019 R_{\odot}$, stellar mass $M_{*}=1.069 \pm 0.048 \quad M_{\odot}$, and surface gravity $\log g=4.399 \pm 0.012$. Additionally, Huber et al. (2013) use Stellar Parameter Classification (SPC, Buchhave et al. 2012, 2014) on spectra from Keck-HIRES (Vogt et al. 1994), the TRES spectrograph on the $1.5 \mathrm{~m}$ at Whipple Observatory (Furesz 2008) and the Tull spectrograph on the $2.5 \mathrm{~m}$ at McDonald Observatory (Tull et al. 1995), to obtain a metallicity of $[\mathrm{Fe} / \mathrm{H}]=0.350 \pm 0.101$ and effective temperature $T_{\text {eff }}=5739 \pm 75 \mathrm{~K}$. McQuillan et al. (2013) searched for the stellar rotation period of KOI-273 in the autocorrelation function of the Kepler photometry and were unable to detect it.

In this paper we measure the mass of KOI-273.01, determine a spectroscopic orbit for an additional Jovian planet and constrain the orbit of a widely separated companion, by analyzing the 2014 and 2015 seasons of HARPS-N radial velocities and several seasons of HIRES radial velocities. As this work confirms the planetary nature of KOI-273.01, we adopt the convention of referring to it as Kepler-454b, and the two more distant companions as Kepler-454c and Kepler-454d, respectively. In Sections 2 and 3, we analyze the spectroscopic and asteroseismic parameters of the star, respectively. In Section 4 we model the Kepler transit photometry. In Section 5, we discuss our RV observations and the data reduction process. In Section 6, we develop a RV model to describe the Kepler454 system. In Section 7, we conclude with a discussion of the
Table 1

Stellar Parameters of Kepler-454

\begin{tabular}{lcc}
\hline \hline Parameter & Value and $1 \sigma$ Errors & References \\
\hline R.A. & $19^{\mathrm{h}} 09^{\mathrm{m}} 54^{\mathrm{s}} .841$ & Høg et al. (2000) \\
decl. & $+38^{\mathrm{d}} 13^{\mathrm{m}} 43^{\mathrm{s} .95}$ & Høg et al. (2000) \\
Kepler magnitude & 11.457 & Borucki et al. $(2011)$ \\
$V$ magnitude & 11.57 & Høg et al. (2000) \\
$K_{s}$ magnitude & 9.968 & Cutri et al. (2003) \\
$\log g$ & $4.395_{-0.055}^{+0.077}$ & this work \\
$R_{*}\left(R_{\odot}\right)$ & $1.066^{\prime} \pm 0.012$ & this work \\
$M_{*}\left(M_{\odot}\right)$ & $1.028_{-0.03}^{+0.04}$ & this work \\
$\rho_{*}\left(\mathrm{~g} \mathrm{~cm}{ }^{-3}\right)$ & $1.199{ }_{-0.014}^{+0.015}$ & this work \\
Age $(\mathrm{Gyr})$ & $5.25_{-1.39}^{+1.41}$ & this work \\
$T_{\text {eff }}(\mathrm{K})$ & $5687 \pm 49$ & this work, SPC \\
{$[\mathrm{m} / \mathrm{H}]$} & $0.32 \pm 0.08$ & this work, SPC \\
$v$ sin $i\left(\mathrm{~km} \mathrm{~s}^{-1}\right)$ & $<2.4$ & this work, SPC \\
$\log g$ & $4.37 \pm 0.06$ & this work, MOOG \\
$T_{\text {eff }}(\mathrm{K})$ & $5701 \pm 34$ & this work, MOOG \\
{$[\mathrm{Fe} / \mathrm{H}]$} & $0.27 \pm 0.04$ & this work, MOOG \\
$\xi_{\mathrm{t}}\left(\mathrm{km} \mathrm{s}{ }^{-1}\right)$ & $0.98 \pm 0.07$ & this work, MOOG \\
\hline
\end{tabular}

mass measurement of Kepler-454b in the context of the bulk densities of small planets.

\section{SPECTROSCOPIC ANALYSIS OF STELLAR PARAMETERS}

We used SPC to derive the stellar parameters of the host star from high-resolution, high signal-to-noise ratio (S/N) HARPS$\mathrm{N}$ spectra, with an average $\mathrm{S} / \mathrm{N}$ per resolution element of 85 . More details on these observations are provided in Section 5. We ran SPC both with all parameters unconstrained and with the surface gravity constrained to the value determined by asteroseismology (Huber et al. 2013, $\log g=4.40 \pm 0.01$ ). The surface gravity from the unconstrained SPC analysis, $\log g=4.37 \pm 0.10$, is in close agreement with the asteroseismic value. For the final parameters, we constrained the surface gravity to the value from asteroseismology. The weighted mean of the SPC results from the individual spectra yielded $T_{\text {eff }}=5687 \pm 50 \mathrm{~K},[\mathrm{~m} / \mathrm{H}]=0.32 \pm 0.08$ and $v \sin$ $i<2.4 \mathrm{~km} \mathrm{~s}^{-1}$.

We also determined the atmospheric parameters using the line analysis code MOOG (Sneden 1973, version 2014) and a Kurucz model atmosphere with the new opacity distribution function (ODFNEW; Castelli \& Kurucz 2004; Kurucz 1992), as done in Dumusque et al. (2014). We measured the equivalent widths of iron spectral lines on a coadded spectrum of $\mathrm{S} / \mathrm{N} \simeq 400$ using ARES v2 with automatic continuum determination (Sousa et al. 2015). We used the linelist from Sousa et al. (2011), with the log gf modified to account for the solar iron abundance adopted in MOOG $(\log \epsilon(\mathrm{Fe})=7.50)$. We obtained $T_{\text {eff }}=5701 \pm 34 \mathrm{~K}$, surface gravity $\log g=$ $4.37 \pm 0.06$, microturbulent velocity $\xi_{\mathrm{t}}=0.98 \pm 0.07 \mathrm{~km} \mathrm{~s}^{-1}$, and iron abundance $[\mathrm{Fe} / \mathrm{H}]=+0.27 \pm 0.04$, where the errors include the dependence of the parameters on temperature. Constraining the gravity to the asteroseismology value did not produce any change in the other parameters. Both sets of stellar parameters are summarized in Table 1.

We used the relationship in Mamajek \& Hillenbrand (2008) to estimate the rotational period of Kepler-454 from $\log R_{\mathrm{HK}}^{\prime}$ and $B-V=0.81$, resulting in a value of $44.0 \pm 4.4$ days. This estimate is consistent with the minimum rotation period of 

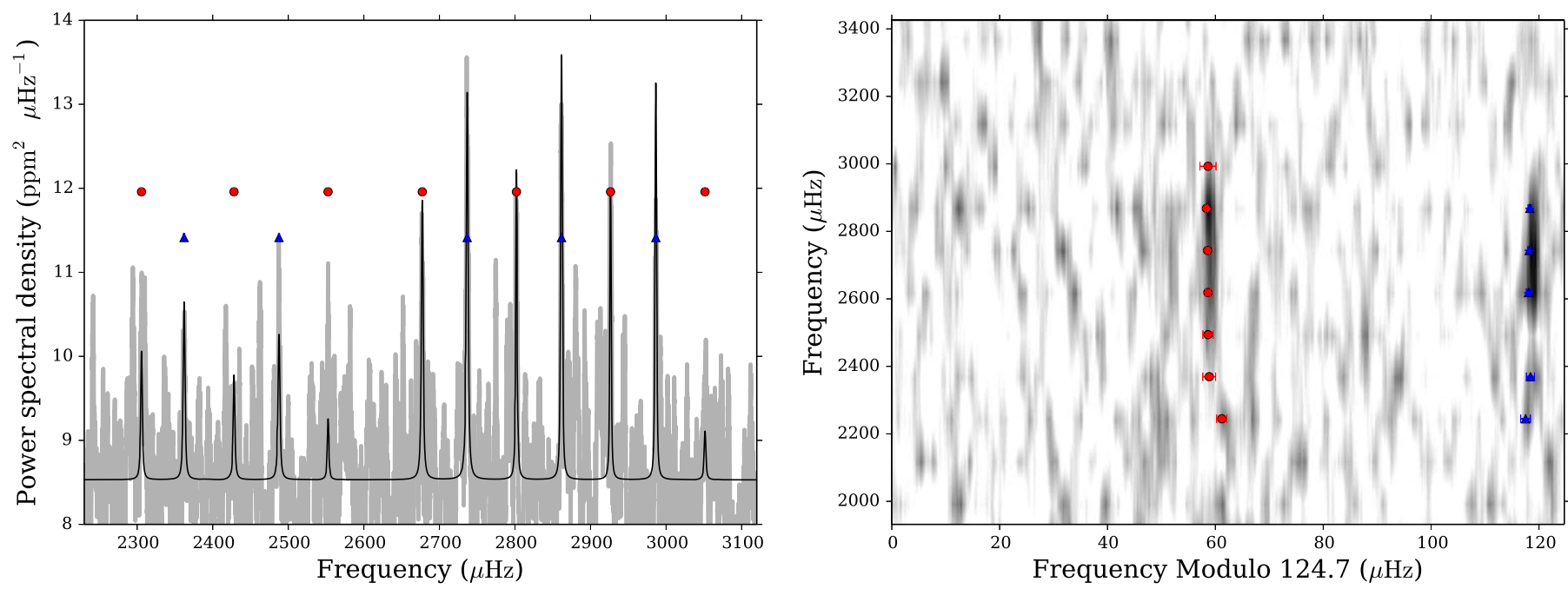

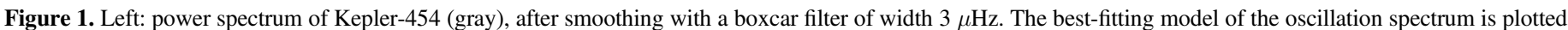

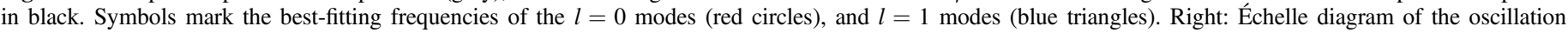

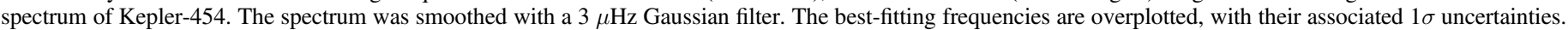

23 days we would obtain by combining the stellar radius and upper limit on the projected rotational velocity from SPC, if we assumed that the rotational axis is aligned with the orbital plane of the inner planet.

\section{ASTEROSEISMIC ESTIMATION OF FUNDAMENTAL STELLAR PROPERTIES}

\subsection{Estimation of Individual Oscillation Frequencies}

The detection of solar-like oscillations was first reported in Kepler-454 by Huber et al. (2013). That study used just one asteroseismic measured parameter-the average large frequency separation, $\Delta \nu$, between overtones-to model the star. Here, we perform a more detailed analysis, using frequencies of 12 individual modes spanning 7 radial overtones.

The results come from the analysis of Kepler short-cadence data, which are needed to detect the short-period oscillations shown by the star. Kepler- 454 was observed in short-cadence in Kepler observing quarters 4, 6 through 12, and 15 through 17. A light curve was prepared for asteroseismic analysis using the KASOC filter (Handberg \& Lund 2014). This mitigates the planetary transits, and minimizes the impact of instrumental artifacts and noise.

The left panel of Figure 1 shows the power spectrum of the prepared light curve after smoothing with a $3 \mu \mathrm{Hz}$ wide boxcar filter. The plotted range shows peaks due to acoustic (pressure, or $p$ ) modes of high radial order. The right panel of Figure 1 shows the échelle diagram, made by dividing the power spectrum into frequency segments of length equal to $\Delta \nu$. When arranged vertically, in order of ascending frequency, the diagram shows clear ridges, comprising overtones of each angular degree, $l$.

Despite the low $\mathrm{S} / \mathrm{N}$, it was possible to extract estimates of individual frequencies. Mode identification was performed by noting that the ridge in the center of the échelle diagram invariably corresponds to $l=0$ in stars with such values of $\Delta \nu$ (White et al. 2011, 2012).

We produced a set of initial frequencies using a matched filter response fit to an asymptotic relation for the $p$-mode
Table 2

Estimated Oscillation Frequencies of Kepler-454 $(\mu \mathrm{Hz})$

\begin{tabular}{lc}
\hline \hline$l=0$ & $l=1$ \\
\hline $2305.79 \pm 0.94$ & $2362.12 \pm 0.90$ \\
$2428.16 \pm 1.19$ & $2487.73 \pm 0.75$ \\
$2552.64 \pm 0.95$ & $\cdots$ \\
$2677.31 \pm 0.43$ & $2736.76 \pm 0.39$ \\
$2801.95 \pm 0.30$ & $2861.59 \pm 0.26$ \\
$2926.45 \pm 0.22$ & $2986.41 \pm 0.31$ \\
$3051.43 \pm 1.49$ & $\cdots$ \\
\hline
\end{tabular}

spectrum, which located frequencies along the ridges in the échelle diagram (Gilliland et al. 2011). We then used the "peak bagging" methodology described by Davies et al. (2015) to extract the individual frequencies. We performed a Markov Chain Monte Carlo (MCMC) optimization fit of the power spectrum, with the oscillation spectrum modeled as a sum of Lorentzian profiles. The adopted procedures have recently been used to fit 33 Kepler planet-hosting stars, and full details are given in Davies et al. (2015).

The estimated frequencies are plotted in both panels of Figure 1 , with the $l=0$ modes shown as red circles, and $l=1$ modes as blue triangles. Some weak power is present in the $l=2$ ridge, but estimates of the $l=2$ frequencies are marginal and we have elected not to use them in our modeling. Bestfitting frequencies and equivalent $1 \sigma$ uncertainties are given in Table 2. The reported frequencies have been corrected for the Doppler shift caused by the motion of the star relative to the observer (here, a shift of $-71 \mathrm{~km} \mathrm{~s}^{-1}$ ), using the prescription in Davies et al. (2014). The correction for the most prominent modes is about $0.6 \mu \mathrm{Hz}$.

Finally, it is worth noting that the best-fitting frequencies very closely match the observed low- $l$ solar $p$-mode frequencies after the latter have been scaled homologously (Bedding \& Kjeldsen 2010) by the ratio of the respective average large separations. This is not surprising given the similarity in mass and age to the Sun, and also confirms that we have correctly assigned the $l$ values. 


\subsection{Detailed Modeling of the Host Star}

Stellar properties were determined by fitting the spectroscopic constraints and extracted oscillation frequencies to several sets of models using different techniques. These techniques are based on the use of the individual frequencies or combinations of frequencies to determine the best-fitting model and statistical uncertainties. Here, we used four different techniques, in the same configurations that were employed to model 33 Kepler exoplanet host stars (Silva Aguirre et al. 2015): the BAyesian STellar Algorithm (BASTA), coupled to grids of GARSTEC (Weiss \& Schlattl 2008) stellar evolutionary models; the ASTEC Fitting method and the Asteroseismic Modeling Portal, both coupled to ASTEC (Christensen-Dalsgaard 2008) models; and the Yale MonteCarlo Method, coupled to YREC (Demarque et al. 2008) models. Detailed descriptions of the techniques were given by Silva Aguirre et al. (2015).

We found excellent agreement, at the level of precision of the data, in the stellar properties estimated by the different techniques. The final properties listed in Table 1 are those obtained from combining grids of GARSTEC models with the BASTA code, and include the effects of microscopic diffusion and settling. The quoted uncertainties are the formal, statistical uncertainties.

We additionally consider the combined effect of different systematic uncertainties, namely the use of different sets of asteroseismic observables, different evolutionary and pulsation codes, as well as different choices of input physics. The expected magnitude of these systematic uncertainties (again, see Silva Aguirre et al. 2015) is as follows: $0.3 \%$ (density and radius), $1 \%$ (mass), and 7\% (age) due to the choice of asteroseismic observables (individual frequencies or combination of frequencies); $1 \%$ (density and radius), $2 \%$ (mass), and $9 \%$ (age) due to the choice of technique; $0.8 \%$ (density), $0.7 \%$ (radius), 2.3\% (mass), 9.6\% (age) due to the choice of input physics, and $1.7 \%$ (density), $1.6 \%$ (radius), $3.6 \%$ (mass), and $16.8 \%$ (age) due to the choice of initial helium abundance. The formal uncertainties in Table 1 are derived in the manner of Silva Aguirre et al. (2015) and do not include the systematic effects discussed above.

\section{PHOTOMETRY}

Our photometric analysis of Kepler- 454 is based on 30 months of short cadence data acquired between quarters Q4Q17 (excluding Q5, Q13, and Q14) and includes 76 independent transits. As in Dressing \& Charbonneau (2015), we normalized each transit to remove the effects of long term drifts by fitting a linear trend to the out-of-transit light curve surrounding each transit. Specifically, we used the time intervals 1-3.5 transit durations prior to and following the expected transit center. We divided the flux data by this trend to produce a normalized light curve.

We used a transit model based on Mandel \& Agol (2002) and varied the period $P$, the epoch of transit $T_{0}$, the ratio of the semimajor axis to stellar radius $a / R_{\star}$, the ratio of planet to stellar radius $R_{\mathrm{p}} / R_{\star}$, and the impact parameter $b=a / R_{\star} \cos i$, where $i$ is the orbital inclination. We assumed a circular orbit model and fit for quadratic limb darkening coefficients using the parameterization suggested by Kipping (2013) in which the parameters $q_{1}=\left(u_{1}+u_{2}\right)^{2}$ and $q_{2}=0.5 u_{1} /\left(u_{1}+u_{2}\right)$ are allowed to vary between 0 and 1 .
Table 3

Transit Parameters of Kepler-454

\begin{tabular}{lcc}
\hline \hline Parameter & Median & Error \\
\hline$P$ (days) & 10.57375339 & $7.77 \mathrm{e}-06$ \\
$T_{0}\left(\mathrm{BJD}_{\text {UTC }}\right)$ & 2455008.0675855 & 0.0007718 \\
$a / R_{\star}$ & 18.293 & 1.098 \\
$R_{\mathrm{p}} / R_{\star}$ & 0.02041 & 0.0011 \\
$b$ & 0.9288 & 0.0091 \\
$i(\mathrm{deg})$ & 87.090 & 0.203 \\
$q_{1}$ & 0.489 & 0.094 \\
$q_{2}$ & 0.517 & 0.330 \\
$u_{1}$ & 0.709 & 0.480 \\
$u_{2}$ & -0.023 & 0.453 \\
$R_{\mathrm{p}}\left(R_{\oplus}\right)$ & 2.37 & 0.13 \\
\hline
\end{tabular}

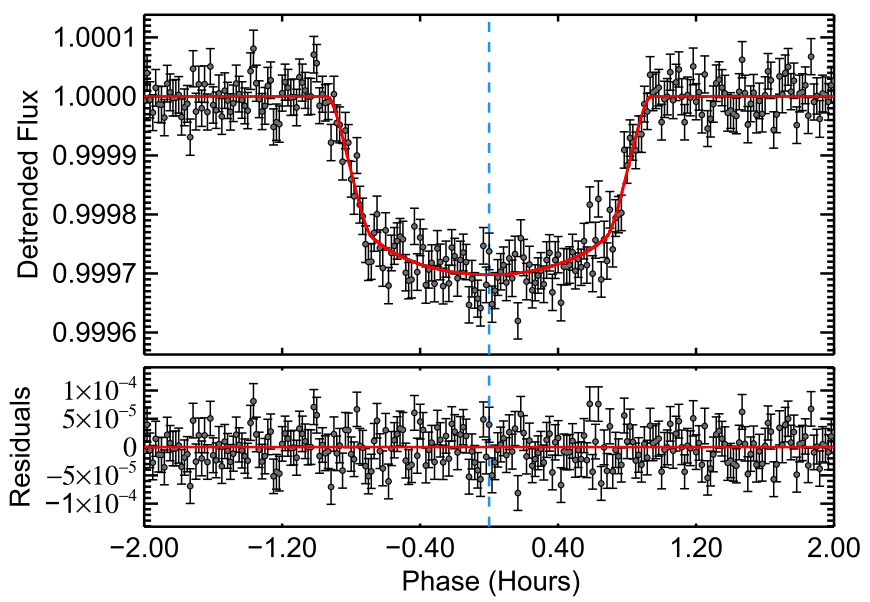

Figure 2. Top: detrended, normalized, phased short cadence observations of Kepler-454 from transits binned to one-minute intervals (data points with errors). The adopted transit model is shown in red and the transit center is marked by the dashed blue line. Bottom: residuals to the transit fit.

We constrained the transit parameters by performing a MCMC analysis with a Metropolis-Hastings acceptance criterion (Metropolis et al. 1953), starting with the initial parameters provided by Batalha et al. (2013). We initialized the chains with starting positions set by perturbing the solution of the preliminary fit by up to $5 \sigma$ for each parameter. The step sizes for each parameter were adjusted to achieve an acceptance fraction between $10 \%$ and $30 \%$.

We ran each chain for a minimum of $10^{4}$ steps and terminated them once each parameter had obtained a GelmanRubin reduction factor $\hat{R}<1.03$. This statistic compares the variance of a parameter in an individual Markov chain to the variance of the mean of that parameter between different chains (Gelman \& Rubin 1992; Ford 2005) and is used to identify chains that have not yet converged. A value of $\hat{R}>1.1$ suggests that a chain has not converged (Gilks et al. 1995). While lower values of $\hat{R}$ are not a definite indicator of convergence, we select a stopping criteria of $\hat{R}<1.03$ as a balance between probability of convergence and computational efficiency.

We removed all steps prior to the step at which the likelihood first exceeded the median likelihood of the chain, to account for burn-in. We merged the chains and used the median values of each parameter as the best-fit value. We chose the error bars to be symmetric and span the $68 \%$ of values closest 
Table 4

HARPS-N Radial Velocity Measurements of Kepler- $454^{\mathrm{a}}$

\begin{tabular}{|c|c|c|c|c|c|c|c|}
\hline $\begin{array}{l}\text { BJD }_{\text {UTC }} \\
-2400000\end{array}$ & $\begin{array}{l}\text { Radial Velocity } \\
\qquad\left(\mathrm{m} \mathrm{s}^{-1}\right)\end{array}$ & $\begin{array}{c}\sigma_{\mathrm{RV}} \\
\left(\mathrm{m} \mathrm{s}^{-1}\right)\end{array}$ & $\begin{array}{l}\text { Bisector Span } \\
\left(\mathrm{m} \mathrm{s}^{-1}\right)\end{array}$ & $\begin{array}{l}\log R_{\mathrm{HK}}^{\prime} \\
\quad(\operatorname{dex})\end{array}$ & $\begin{array}{l}\sigma \log R_{\mathrm{HK}}^{\prime} \\
\quad(\mathrm{dex})\end{array}$ & $\begin{array}{l}t_{\exp } \\
(\mathrm{s})\end{array}$ & Notes \\
\hline 56813.668879 & -71408.25 & 1.42 & -29.41 & -5.03 & 0.02 & 1800 & \\
\hline 56814.513734 & -71408.84 & 1.78 & -40.04 & -5.01 & 0.03 & 1800 & \\
\hline 56815.522009 & -71408.40 & 1.55 & -26.38 & -5.03 & 0.02 & 1800 & \\
\hline 56816.591592 & -71408.43 & 1.58 & -29.14 & -5.02 & 0.03 & 1800 & \\
\hline 56828.676554 & -71400.04 & 1.75 & -25.51 & -5.05 & 0.03 & 1800 & \\
\hline
\end{tabular}

Note.

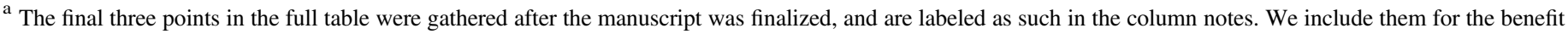
of future workers but they were not included in our analysis.

(This table is available in its entirety in machine-readable form.)

to the best-fit value. These values are shown in Table 3 and the fit to the short-cadence observations is shown in Figure 2.

Our best-fit orbital period and time of transit agree well with those reported in the Cumulative Kepler Object of Interest table on the NASA Exoplanet Archive ${ }^{23}$ as of 2015 May 19, but we found a significantly larger value for the impact parameter. In contrast to the previously reported value of $b=0.1_{-0.0999}^{+0.3703}$, we find $b=0.929 \pm 0.009$, which is $2.2 \sigma$ discrepant from the previous value. Our results for $a / R_{\star}$ and $R_{\mathrm{p}} / R_{\star}$ are also in disagreement with the KOI table results: we estimate $a / R_{\star}=18.293 \pm 1.098 \quad$ and $\quad R_{\mathrm{p}} / R_{\star}=0.0204 \pm 0.001$ whereas the cumulative KOI list reports $a / R_{\star}=48.17 \pm 3.6$ and $R_{\mathrm{p}} / R_{\star}=0.015743_{-0.000202}^{+0.0005}$.

The larger value for $R_{\mathrm{p}} / R_{\star}$ translates directly into an inferred planetary radius that is significantly larger than the $R_{\mathrm{p}}=1.86 \pm 0.03 R_{\oplus}$ value reported in the cumulative $\mathrm{KOI}$ table. Combining the uncertainty in $R_{\mathrm{p}} / R_{\star}$ and the uncertainty on the stellar radius $\left(1.06568 \pm 0.01200 R_{\odot}\right)$, we find a planet radius of $2.37 \pm 0.13 R_{\oplus}$. Given that our values are consistent with those found by Sliski \& Kipping (2014) in an independent analysis of the Kepler-454b short cadence data, we attribute the disagreement between the results of our analysis and the values reported in the Cumulative KOI table to the degraded ability of analyses based on long cadence data to tightly constrain the impact parameter. In addition, the use of fixed limb darkening parameters for the fit reported in the Cumulative KOI Table may have contributed to the discrepancy.

In addition to revising the transit photometry for Kepler$454 \mathrm{~b}$, we searched for transits due to additional companions using a Box-fitting Least Squares analysis (Kovács et al. 2002), but none were found. We also investigated the possibility that the transit times of Kepler-454b might differ from a linear ephemeris due to light travel time effects or perturbations from the non-transiting companions. Starting with the best-fit solution from the MCMC fit to the short cadence data, we fit each transit event separately allowing the transit center to vary but holding $a / R_{\star}, R_{\mathrm{p}} / R_{\star}$, and $b$ constant. Although the individual times of transit shifted by -10 to +8 minutes, these were consistent with the timing precision for individual transits and we did not find evidence for correlated shifts in the transit times. We therefore adopted a linear ephemeris for Kepler454b (see Table 3).

There is a small possibility that the transit of Kepler-454 is a false positive, and the transit signal is due to an eclipsing binary

\footnotetext{
23 http://exoplanetarchive.ipac.caltech.edu/cgi-bin/TblView/nph-tblView? app $=$ ExoTbls\&config $=$ cumulative
}

(either physically bound, or a change alignment on the sky). Morton \& Johnson (2011) estimate the false positive probability of the Kepler-454 system at about $1 \%$ based on Galactic structure models. Fressin et al. (2013) simulated blends of eclipsing binaries and estimated the fraction that would occur and pass Kepler candidate vetting procedures; those authors estimated a false positive rate of $6.7 \%$ for planets 2-4 $R_{\oplus}$ (appropriate for Kepler-454b). By observing the change in RV of the star at the orbital period and phase expected from the light curve (see Section 5), we rule out the possibility of a false positive scenario.

\section{RV OBSERVATIONS AND REDUCTION}

\subsection{HARPS-N Observations}

We measured the RV variation of Kepler-454 using the HARPS-N spectrograph on the $3.55 \mathrm{~m}$ Telescopio Nazionale Galileo (TNG) at the Observatorio del Roque de los Muchachos (Cosentino et al. 2012). HARPS-N is a highly precise, high-resolution $(R \simeq 115,000)$, vacuum-stabilized spectrograph, very similar in design to the original HARPS planet hunting instrument at the ESO $3.5 \mathrm{~m}$ (Mayor et al. 2003). Notable improvements include the use of octagonal fibers to improve the scrambling of incoming light and a monolithic $4096 \times 4096$ CCD.

We obtained 55 observations of Kepler-454 during the 2014 observing season and 10 observations during the 2015 season. Most observations were made with a 30 minute exposure time, achieving a mean $\mathrm{S} / \mathrm{N}_{\text {pixel }}{ }^{-1}$ of 48 at $5500 \AA$ and a mean internal precision of $2.2 \mathrm{~m} \mathrm{~s}^{-1}$, estimated by combining photon noise, wavelength calibration noise and instrumental drift. Two of these observations had $\mathrm{S} / \mathrm{N}<30$, corresponding to $>5 \mathrm{~m} \mathrm{~s}^{-1} \mathrm{RV}$ precision, and were not included in the analysis. We observed Kepler-454 without simultaneous wavelength reference, to prevent contamination of the stellar spectrum with light from the ThAr calibration lamp.

The spectra were reduced with the standard HARPS-N pipeline and we measured the radial velocities by using a weighted cross-correlation between the observed spectra and a numerical mask based on the spectrum of a G2V star (Baranne et al. 1996; Pepe et al. 2002). The resulting RV data are listed in Table 4, with their $1 \sigma$ internal uncertainties, epoch in $\mathrm{BJD}_{\mathrm{UTC}}$, and bisector span.

\subsection{HIRES Observations}

We obtained 36 observations of Kepler-454 made with the HIRES spectrograph on the Keck Telescope (Vogt et al. 1994) 
Table 5

HIRES Radial Velocity Measurements of Kepler-454

\begin{tabular}{lcc}
\hline \hline $\begin{array}{l}\text { BJD } \\
-2400000\end{array}$ & $\begin{array}{c}\text { Radial Velocity } \\
\left(\mathrm{m} \mathrm{s}^{-1}\right)\end{array}$ & $\begin{array}{c}\sigma_{\mathrm{RV}} \\
\left(\mathrm{m} \mathrm{s}^{-1}\right)\end{array}$ \\
\hline 55431.809347 & 52.75 & 1.24 \\
55782.924716 & -113.43 & 1.31 \\
55792.863793 & -106.50 & 1.85 \\
55792.895597 & -110.48 & 1.40 \\
55797.795127 & -99.82 & 1.26 \\
\hline
\end{tabular}

(This table is available in its entirety in machine-readable form.)

through collaboration with the California Planet Search team. These observations were obtained between 2010 August and 2014 Dececember, with a mean S/N of 165 and a typical internal precision of $1.4 \mathrm{~m} \mathrm{~s}^{-1}$. The $\mathrm{RV}$ measurements were calibrated using an iodine absorption cell (Butler et al. 1996), and their reduction is described in Marcy et al. (2014). They are listed in Table 5, with their epoch in BJD $_{\text {UTC }}$ and their $1 \sigma$ internal errors. These observations were made as part of NASA's Kepler Key Project follow-up program, and as such all raw and reduced spectra are made available to the public in the Keck Observatory Archive, and the RV measurements will be made available on CFOP.

\section{ANALYSIS OF RADIAL VELOCITY MEASUREMENTS}

The combined HARPS-N and HIRES measurements of Kepler-454 show two Keplerian orbits and a long-term trend consistent with an additional companion. We join these data using a single offset term for the HIRES observations and model the radial velocities as a sum of two Keplerian signals,

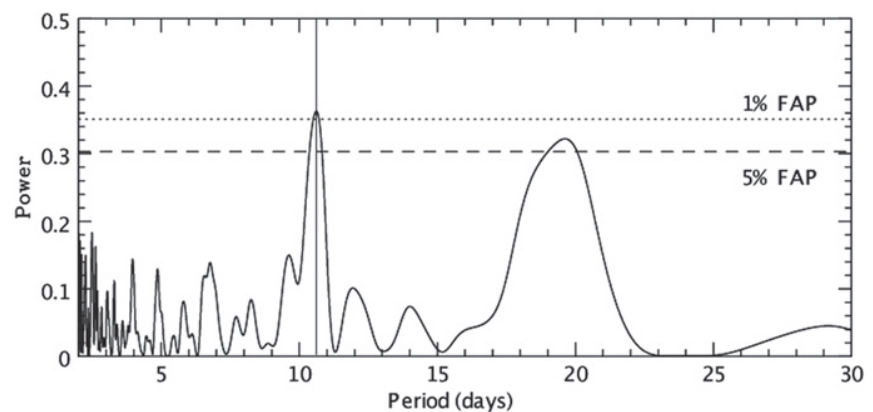

Figure 3. Periodogram of the 2014 HARPS-N RV measurements of Kepler454 , after removal of the signal from the outer companions. The vertical line marks the period of Kepler- $454 \mathrm{~b}$ and the $1 \%$ and 5\% FAP levels are shown as dashed lines.

plus a linear trend:

$$
\begin{aligned}
\mathcal{M}\left(t_{i}\right)= & \gamma+\mathrm{RV}_{\mathrm{off}}+\beta t_{i} \\
& +\sum_{j=1}^{2} K_{j}\left[\cos \left(\theta_{j}\left(t_{i}, T_{p, j}, P_{j}, e_{j}\right)+\omega_{j}\right)+e_{j} \cos \left(\omega_{j}\right)\right]
\end{aligned}
$$

where $\mathrm{RV}_{\text {off }}$ is the offset of the HIRES observations from the HARPS-N observations (for the HIRES data, the velocities of each observation are estimated relative to a chosen epoch and hence are relative; for the HARPS-N data, the velocities are measured relative to a theoretical template tied to laboratory rest wavelengths), $\gamma$ is the systemic velocity of Kepler-454 and $\beta$ is the slope of the linear trend due to a long-period companion. Individual orbits $j$ are characterized by their semiamplitude $K$, period $P$, time of periastron passage $T_{\mathrm{p}}$,

Table 6

\begin{tabular}{|c|c|c|c|c|}
\hline Parameter & $\begin{array}{l}\text { Best Fit } \\
\text { Median }\end{array}$ & $\begin{array}{l}\text { Best Fit } \\
1 \sigma \text { Error }\end{array}$ & $\begin{array}{c}\text { Eccentric Fit } \\
\text { Median }\end{array}$ & $\begin{array}{c}\text { Eccentric Fit } \\
1 \sigma \text { Error }\end{array}$ \\
\hline \multicolumn{5}{|c|}{ Kepler-454b } \\
\hline$P$ (days) & 10.573753 & $7.5 \times 10^{-6}$ & 10.573753 & $7.5 \times 10^{-6}$ \\
\hline$T_{0}\left(\mathrm{BJD}_{\mathrm{UTC}}\right)$ & 2455008.06758 & 0.00076 & 2455008.06758 & 0.00076 \\
\hline$e$ & 0.0 & $\ldots$ & 0.23 & 0.13 \\
\hline$\omega(\operatorname{deg})$ & $\ldots$ & $\ldots$ & 341.6 & 52.8 \\
\hline$K\left(\mathrm{~m} \mathrm{~s}^{-1}\right)$ & 1.96 & 0.38 & 2.16 & 0.43 \\
\hline$m_{\mathrm{p}}\left(M_{\oplus}\right)$ & 6.84 & 1.40 & 7.24 & 1.40 \\
\hline$a(\mathrm{AU})$ & 0.0954 & 0.0012 & 0.0954 & 0.0012 \\
\hline \multicolumn{5}{|c|}{ Kepler-454c } \\
\hline$P$ (days) & 523.90 & 0.70 & 523.89 & 0.78 \\
\hline$T_{\mathrm{p}}\left(\mathrm{BJD}_{\mathrm{UTC}}\right)$ & 2454892 & 26 & 2454897 & 27 \\
\hline$e$ & 0.0214 & 0.0077 & 0.0199 & 0.0064 \\
\hline$\omega(\operatorname{deg})$ & 337.4 & 17.4 & 341.3 & 18.2 \\
\hline$K\left(\mathrm{~m} \mathrm{~s}^{-1}\right)$ & 110.44 & 0.96 & 110.65 & 0.99 \\
\hline$m_{\mathrm{p}} \sin i\left(M_{J}\right)$ & 4.46 & 0.12 & 4.47 & 0.12 \\
\hline$a \sin i(\mathrm{AU})$ & 1.286 & 0.0166 & 1.286 & 0.0166 \\
\hline \multicolumn{5}{|c|}{ Kepler-454 System } \\
\hline$\gamma\left(\mathrm{m} \mathrm{s}^{-1} \mathrm{~d}^{-1}\right)$ & -71322.23 & 0.61 & -71321.91 & 0.61 \\
\hline$d v / d t\left(\mathrm{~m} \mathrm{~s}^{-1} \mathrm{~d}^{-1}\right)$ & 0.0429 & 0.0016 & 0.0431 & 0.0016 \\
\hline HIRES offset $\left(\mathrm{m} \mathrm{s}^{-1} \mathrm{~d}^{-1}\right)$ & -71324.77 & 0.95 & -71324.85 & 0.95 \\
\hline
\end{tabular}

RV Parameters of Kepler-454

Epoch of Fit $\left(\right.$ BJD $\left._{\text {UTC }}\right)=2456847.8981528$ 

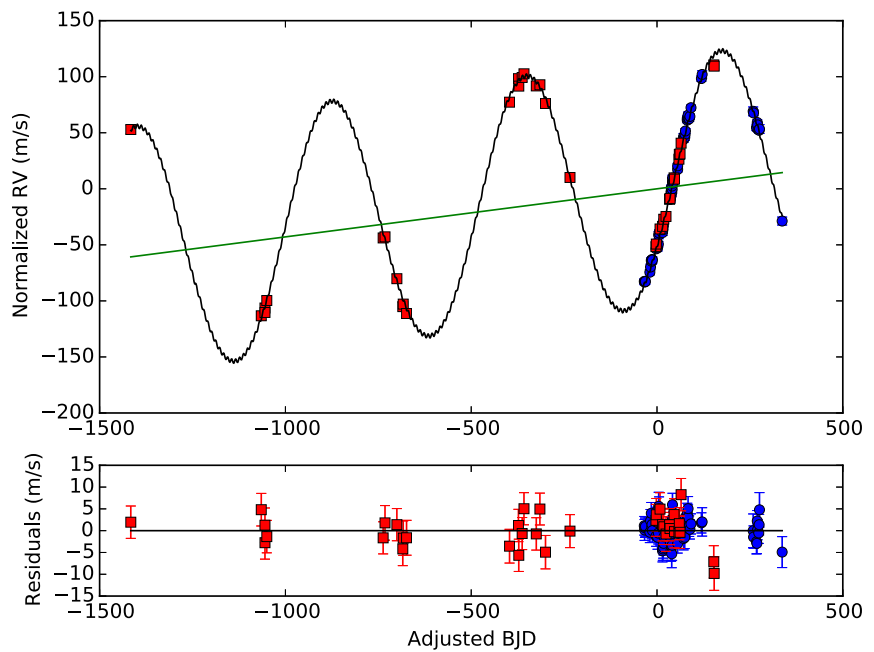

Figure 4. Top: RV observations from HARPS-N (blue circles) and KeckHIRES (red squares), along with the best-fit circular orbit + Keplerian orbit + trend model (black line) and trend only (green line). The error bars are the internal measurement errors and jitter combined in quadrature. Bottom: the residuals to the best-fit model are shown (blue circles and red squares).
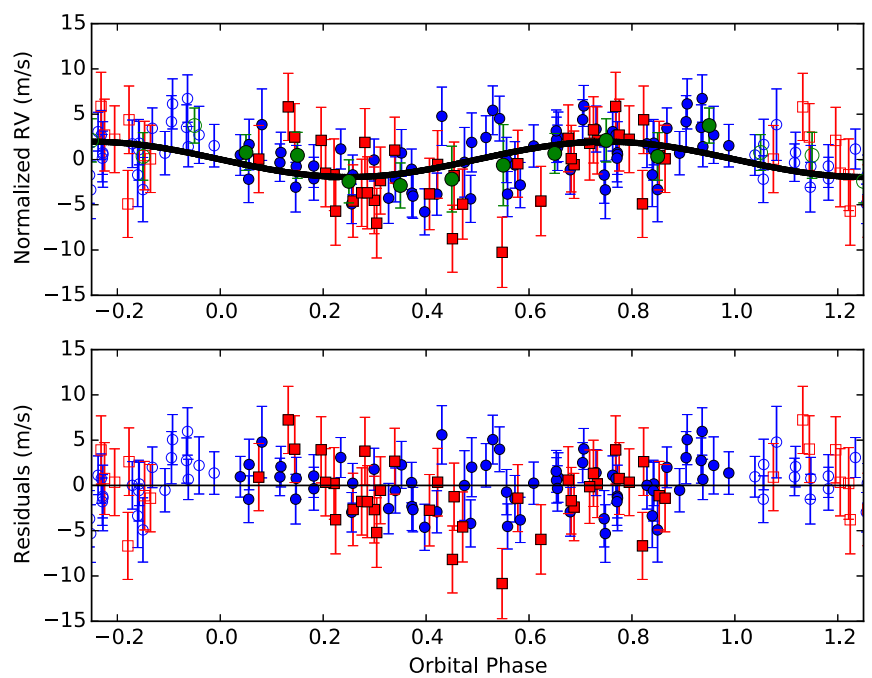

Figure 5. Top: phased RV observations from HARPS-N (blue circles) and Keck-HIRES (red squares) after subtracting the outer orbits. The best-fit circular model for the inner planet is shown as a black line. The error bars are the internal measurement errors and jitter combined in quadrature. The large green circles show the observations binned at intervals of 0.1 orbital phase. Open symbols denote repeated phases. Bottom: the residuals to the best-fit model are shown (blue circles and red squares).

eccentricity $e$, and argument of periastron $\omega$. The function $\theta$ is the true anomaly of the planet at epoch $t_{i}$. We included the time of reference transit $T_{0}$ as an additional constraint relating $\omega_{j}, T_{p, j}$ and $e_{j}$. We use the convention for circular orbits that $\omega=90^{\circ}$, such that $T_{\mathrm{p}}=T_{0}$ and use the standard relationships for eccentric orbits:

$$
\begin{gathered}
\theta_{0}=90^{\circ}-\omega \\
\tan \left(\frac{\theta_{0}}{2}\right)=\left(\frac{1+e}{1-e}\right)^{1 / 2} \tan \left(\frac{E_{0}}{2}\right) \\
T_{0}-T_{\mathrm{p}}=E_{0}-e \sin \left(E_{0}\right) \frac{P}{2 \pi}
\end{gathered}
$$

where $\theta_{0}$ is the true anomaly of transit time and $E_{0}$ is the eccentric anomaly of transit time (Danby 1988).

We considered circular and eccentric orbits for both planets. We fit an initial solution using a Levenberg-Marquardt minimization algorithm ${ }^{24}$ and then these parameters were used as input to emcee, an Affine Invariant MCMC ensemble sampler package (Foreman-Mackey et al. 2013). We initialized 200 chains with starting positions selected by perturbing each free parameter of the LM solution by an amount drawn from a tight Gaussian distribution with width $10^{-6}$ times the magnitude of that parameter, consistent with the suggested initialization of emcee. We set uniform priors on all parameters except $P_{1}$ and $T_{0,1}$, for which we use Gaussian priors determined by the best-fit values from Section 4 . We force $K$ to be non-negative and we transformed variables $e$ and $\omega$ to $\sqrt{e} \cos (\omega)$ and $\sqrt{e} \sin (\omega)$ to improve the convergence of low-eccentricity solutions.

As in Dumusque et al. (2014), we model the stellar signal as a constant jitter term $\sigma_{j}$ and use the following likelihood:

$$
\mathcal{L}=\prod_{i=1}^{N}\left(\frac{1}{\sqrt{2 \pi\left(\sigma_{i}^{2}+\sigma_{j}^{2}\right)}} \exp \left[-\frac{\left(\operatorname{RV}\left(t_{i}\right)-\mathcal{M}\left(t_{i}\right)\right)^{2}}{2\left(\sigma_{i}^{2}+\sigma_{j}^{2}\right)}\right]\right)
$$

where $\mathrm{RV}\left(t_{i}\right)$ is the observed $\mathrm{RV}$ at time $t_{i}$ and $\mathcal{M}$ is the model. The stellar jitter noise $\sigma_{j}$ is assumed to be constant and $\sigma_{i}$ is the internal noise for each epoch. The stellar jitter is forced to be positive and allowed to have different values for the HARPS-N and HIRES datasets.

We check for convergence by computing the Gelman-Rubin reduction factor and determined the chains to have converged once all variables had attained $\hat{R}<1.03$. We discard the first $50 \%$ of each chain as the "burn in" stage and combine the remaining portion of the chains. We select the median value of each parameter as the best-fit value and the error bars are chosen to span the $68 \%$ of values closest to the best-fit value. These values are shown in Table 6 . We note that the reported errors on $\gamma$ include only the statistical errors on this fit and not other effects, such as gravitational redshift. The total uncertainty on the systemic velocity is of order $100 \mathrm{~m} \mathrm{~s}^{-1}$.

We consider the null hypothesis that the Kepler-454 system can be described by a linear trend, a marginally eccentric planet in a 524d orbit and Gaussian noise. We compare it to the alternative hypothesis that the Kepler-454 system can be described by those same components, plus a planet in a $10.6 \mathrm{~d}$ circular orbit. We select the model that best describes the data by considering the Bayesian Information Criterion (BIC). We use the BIC values to approximate the Bayes factor between pairs of models, with the stellar jitter terms held fixed at $1.6 \mathrm{~m} \mathrm{~s}^{-1}$ and $3.5 \mathrm{~m} \mathrm{~s}^{-1}$ for HARPS-N and HIRES, respectively. These values are representative of those obtained when the jitter terms were allowed to vary. We find a BIC value of 11.9 in favor of including a circular orbit at 10.6 days. Additionally, we show the periodogram of the 2014 HARPS-N RV measurements in Figure 3, after removing the signal of the outer companions. This series of measurements is most sensitive to short period signals due to its higher cadence and we recover a 10.6 day periodicity with a false-alarm probability (FAP) of just under $1 \%$. This is sufficient evidence that the signal of Kepler-454b is present in the RV measurements.

\footnotetext{
${ }^{24}$ https://github.com/pkgw/pwkit
} 

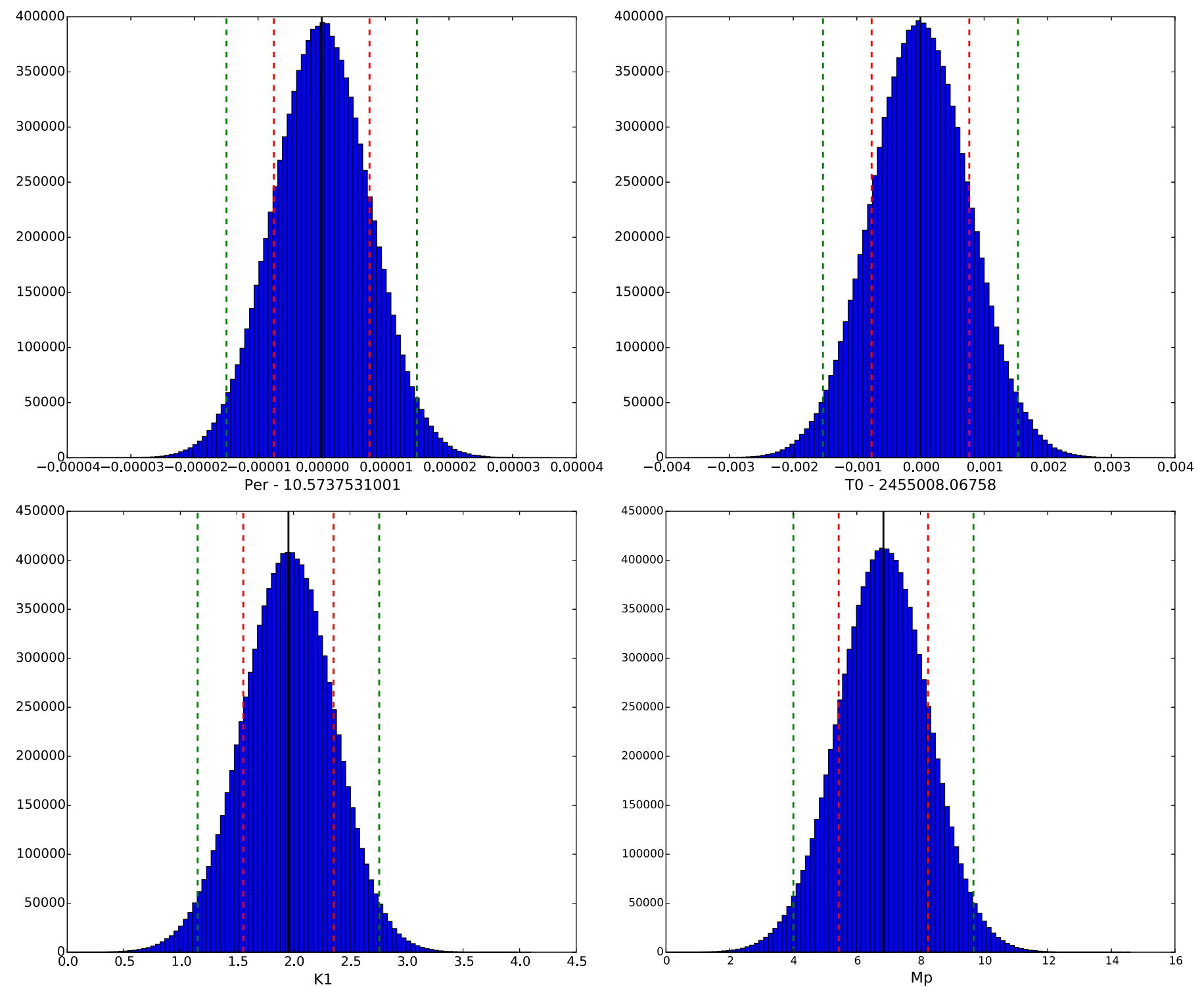

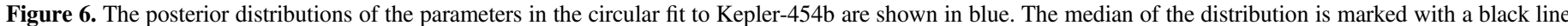
and the dashed red and green lines contain $68.3 \%$ and $95.4 \%$ of the values, respectively.

We next consider whether the orbits are sufficiently modeled as sinusoids. We find a BIC value of 6.9 in favor of a circular orbit for Kepler-454b and a BIC value of 0.9 in favor of a marginally eccentric orbit for Kepler-454c. As the BIC values are an approximation, we performed an independent differential evolution MCMC (DE-MCMC) analysis of the combined RV measurements, and found nearly identical values for the median orbital parameters and their error estimates, for both a circular and eccentric fit to the inner planet. In this case, we used $2 N$ chains, where $N$ is the number of free parameters. We imposed Gaussian priors on the period and transit time of Kepler-454b, and Jeffery's priors on the jitter terms. We stopped the chains after they achieved $\hat{R}<1.01$ and more than 1000 independent draws (Ford 2006; Bonomo et al. 2014). The Bayes factor values were taken directly from the DE-MCMC posterior distributions by using the Truncated Posterior Mixture method (Tuomi \& Jones 2012). We estimated a Bayes factor of $39.2 \pm 2.6$ in favor of an eccentric solution for Kepler-454c and a Bayes factor of $3.5 \pm 0.3$ in favor of an eccentric solution for Kepler-454b. This is strong evidence in favor of an eccentric orbit for Kepler-454c and slight evidence for an eccentric solution for Kepler-454b (Kass \& Raftery 1995).

We adopt the simpler model, using an eccentric outer orbit and circular inner orbit for the best-fit model, obtaining a mass estimate for Kepler- $454 \mathrm{~b}$ of $6.84 \pm 1.40 M_{\oplus}$. The measured RVs and best-fit model are displayed in Figures 4 and 5. The posterior distributions of the orbital parameters of Kepler-454b are shown in Figure 6. When the eccentricity of the inner planet is allowed to vary, we obtain $e=0.23 \pm 0.13$ and a mass estimate of $7.24 \pm 1.40 M_{\oplus}$, consistent with the results from the best-fit solution. We include the parameters of this solution in Table 6 for completeness and show the corresponding posterior distributions in Figure 7. The distribution of residuals to the best-fit solution are shown in Figure 8. The HARPS-N residuals have a median value of $0.01 \mathrm{~m} \mathrm{~s}^{-1}$ and $68 \%$ of the values fall within $2.5 \mathrm{~m} \mathrm{~s}^{-1}$ of the median. For the HIRES residuals, the median is $0.36 \mathrm{~m} \mathrm{~s}^{-1}$ and the distribution is a bit broader, with $68 \%$ of the values fall within $3.5 \mathrm{~m} \mathrm{~s}^{-1}$ of the median. 

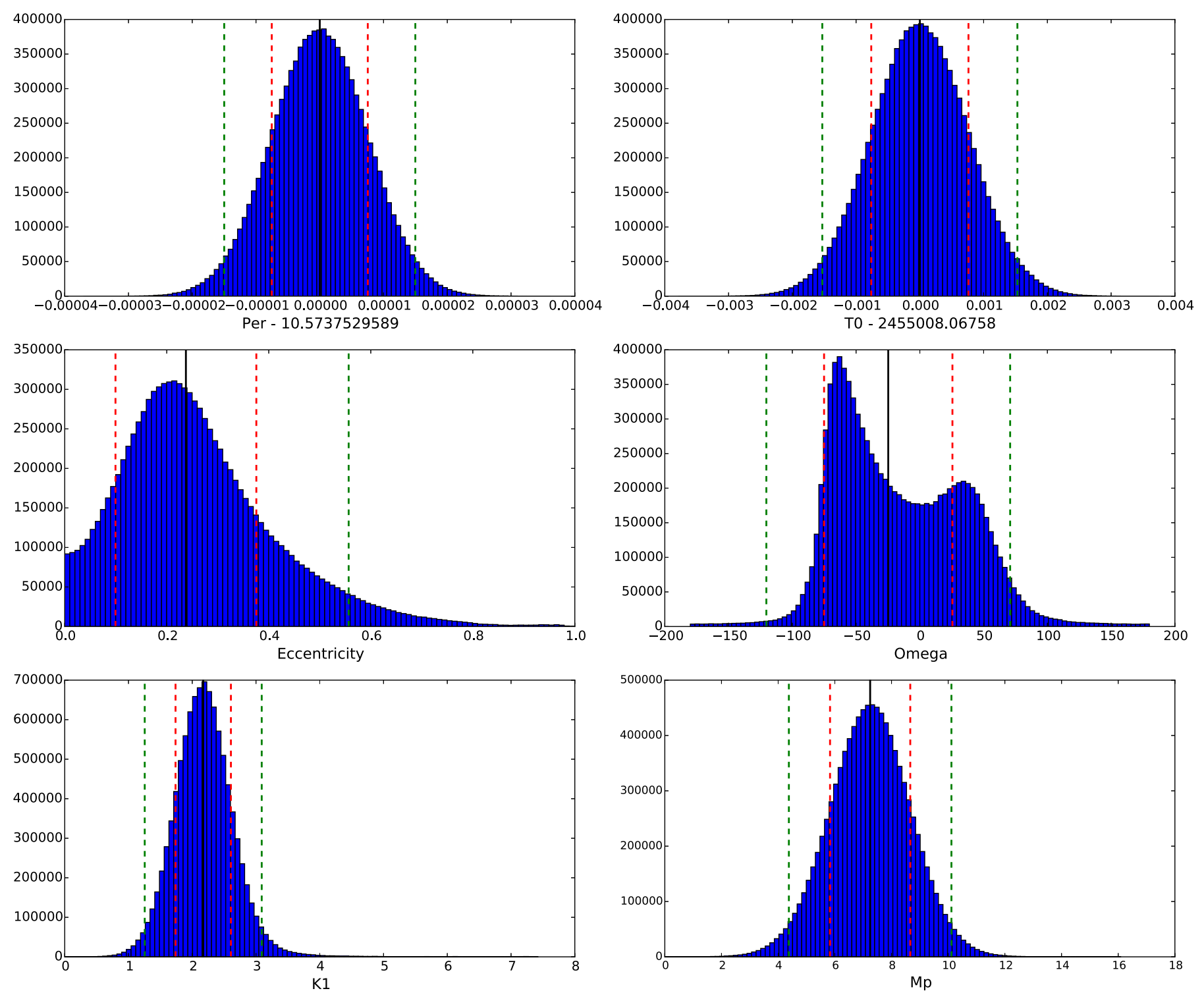

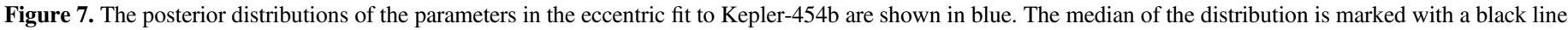
and the dashed red and green lines contain $68.3 \%$ and $95.4 \%$ of the values, respectively.

We calculate the tidal circularization timescale of Kepler$454 \mathrm{~b}$ using the formula of Goldreich \& Souter (1966) for a 6.84 $M_{\oplus}, 2.37 R_{\oplus}$ planet in an $0.095 \mathrm{AU}$ orbit around a $1.028 M_{\odot}$ star. As Kepler-454b is intermediate between a rocky world and a Neptune-like composition, we expect that its tidal quality factor $Q$ may be intermediate as well. If we assume a $Q$ value of 100 , consistent with terrestrial planets in the solar system, the tidal circularization timescale is 440 million years, shorter than the $5.2 \pm 1.4$ billion year age of the system estimated with asteroseismology. If we assume a $Q$ value of 9000 , consistent with Neptune, the circularization timescale is 39 billion years. Obtaining a circularization timescale consistent with the age of Kepler-454 is possible with $Q=1200$, a value that is larger than those seen in terrestrial planets, but smaller than that of Neptune (Zhang \& Hamilton 2008; Henning et al. 2009). Though Kepler-454b may have had enough time to reach a circular orbit, the circularization timescale is not sufficiently well determined to discount an eccentric orbit.

As Kepler-454 has both quality asteroseismology and transit data, we are able to calculate a minimum eccentricity for the inner planet through asterodensity profiling in the manner of Kipping (2014). We compare the stellar density value of $1.199 \pm 0.015 \mathrm{~g} \mathrm{~cm}^{-3}$ obtained through asteroseismology with the stellar density value of $1.04 \pm 0.19 \mathrm{~g} \mathrm{~cm}^{-3}$ implied by a transiting circular orbit and obtain a minimum eccentricity of $0.05 \pm 0.06$, consistent with a circular orbit. Given the ambiguity in whether an eccentric model is necessary for Kepler-454b, we favor the simpler solution but provide parameters for both models in Table 6 .

Characterization of Kepler-454d is difficult, as the period of its orbit is much longer than the timescale of the combined RV measurements. We observe a linear drift rate of $15.7 \pm 0.6 \mathrm{~m} \mathrm{~s}^{-1} \mathrm{yr}^{-1}$ over nearly 5 years. Assuming an edge-on circular orbit, this suggests that Kepler-454d has $P>10$ year, $M \sin (i)>12.1 M_{J}$ and a semimajor axis $>4.7$ AU. Assuming that this solar-like star has an absolute magnitude $M_{V}=4.9$, comparable to the Sun, its distance is approximately $200 \mathrm{pc}$ and the angular separation at the maximum elongation of Kepler-454d is $>0$." 02 . 


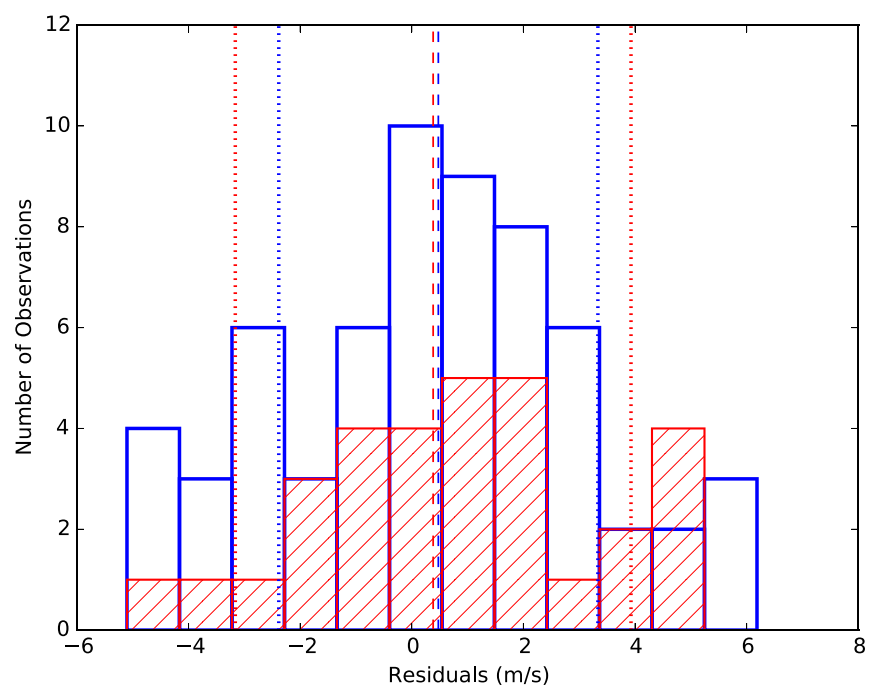

Figure 8. Histogram of best-fit residuals to the HARPS-N observations (blue) and HIRES observations (red). The dashed line marks the medians of each distribution, $0.01 \mathrm{~m} \mathrm{~s}^{-1}$ for HARPS-N and $0.36 \mathrm{~m} \mathrm{~s}^{-1}$ for HIRES. The dotted lines mark symmetric error bars including $68 \%$ of the measurements nearest the medians, $2.5 \mathrm{~m} \mathrm{~s}^{-1}$ for HARPS-N and $3.5 \mathrm{~m} \mathrm{~s}^{-1}$ for HIRES.

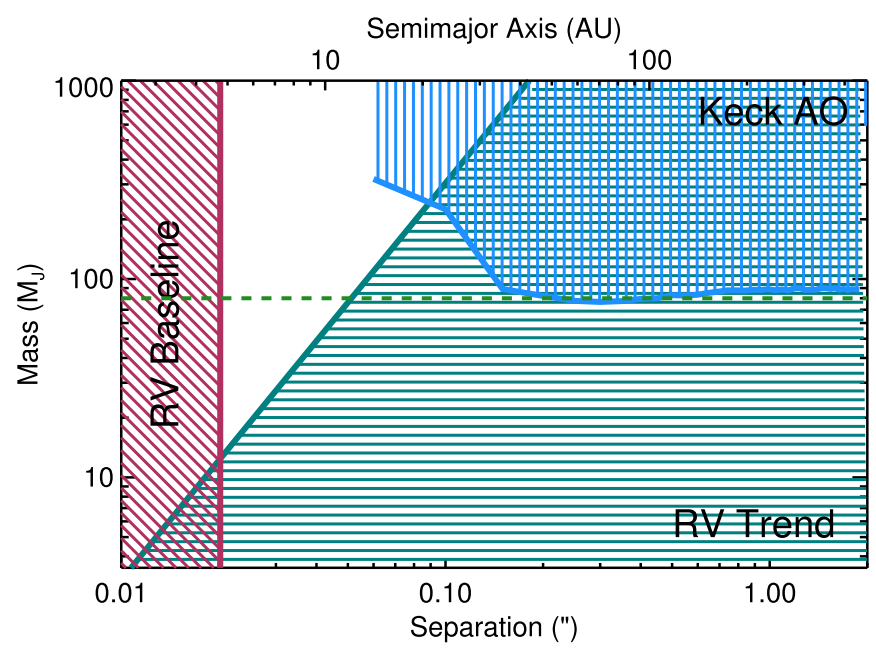

Figure 9. Limits on the mass and separation of Kepler-454d. The baseline of the combined RV observations excludes the maroon region and the amplitude of the RV trend excludes the teal region. The blue region is excluded by the Keck AO observations. These limits assume a circular, coplanar orbit for Kepler-454d. The dashed green line marks $80 M_{J}$, the mass boundary between brown dwarfs and stars.

There are multiple sources of adaptive optics (AO) observations for this target, though none are able to place limits on the brightness of the companion at such a small separation. The most stringent of these are Keck observations made in $B r_{\gamma}$, with a brightness limit at 0 ". 06 of 3.73 mag fainter than the host star (CFOP; D. Ciardi). Assuming that $K_{s}$ magnitude is equivalent to $B r_{\gamma}$ magnitude, Kepler-454d must then be fainter than $K_{s}=13.7$, unless it was not detectable at the time of the AO observations due to orbital geometry. We converted the $K_{s}$ limit into a mass upper limit using the Delfosse et al. (2000) relation and found a value of $300 M_{J}$ for angular separations beyond 0 " 05 . The combined restrictions from the RV and AO data are shown in Figure 9.

Astrometry from the Gaia mission could readily complement our RV measurements. It will provide approximately $16 \mu$ as astrometry for stars with $V=12$ mag (Els et al. 2014). With this precision, Jupiter-sized planet like Kepler-454c would be marginally detectable as a signal of order $30 \mu \mathrm{as}$, providing measurements of inclination and mass. The acceleration due to the outer companion should be readily detectable, further constraining its separation and mass.

While Kepler-454 is an inactive star, with a median $\log R_{\mathrm{HK}}^{\prime}$ value of -5.0 , we consider the possibility of RV variations induced by stellar activity. After removing the signal from the 524 day orbit and linear trend, we compare the RV measurements to the $\log R_{\mathrm{HK}}^{\prime}$ values, and several features of the crosscorrelation function (CCF), including the bisector velocity span, FWHM and the contrast of the CCF. We found no correlations with RV, as shown in Figure 10 and there are no significant periodicities in these indicators. We conclude that it is sufficient to assume a Gaussian noise term in the likelihood function, to model RV variations due to stellar activity.

\section{DISCUSSION}

We present a mass measurement for Kepler-454b of $6.8 \pm 1.4 M_{\oplus}$ and detect two additional non-transiting companions, a planet with a minimum mass of $4.46 \pm 0.12$ $M_{J}$ in a slightly eccentric 524 day orbit and a linear trend consistent with a brown dwarf or low-mass star.

Combining our mass estimate for Kepler-454b with a radius of $2.37 \pm 0.13 \quad R_{\oplus}$, gives a density estimate of $2.76 \pm 0.73 \mathrm{~g} \mathrm{~cm}^{-3}$. Figure 11 shows a mass-radius plot of Kepler-454b along with the several other planets smaller than $2.7 R_{\oplus}$ and with masses measured to better than $20 \%$ precision. There are 12 such planets, including Kepler-454b. Dressing et al. (2015) note that the six planets with radii $<1.6 R_{\oplus}$, as well as Earth and Venus, have very similar uncompressed densities. The recently detected planet HD 219314b also has a comparable density (Motalebi et al. 2015). These planets are consistent with an Earth-like composition, notably the Earth's ratio of iron to magnesium silicates. In contrast, the six planets with radii $2.0 \leqslant R\left(R_{\oplus}\right) \leqslant 2.7$ are not consistent with this rocky composition model. Their lower densities require a significant fraction of volatiles, likely in the form of an envelope of water and other volatiles and/or $\mathrm{H} / \mathrm{He}$. If rocky planets with radii $>1.6 R_{\oplus}$ do exist, they are likely to be more massive and thus easier to detect. If such high density planets continue to be absent as the sample of small planets grows, it would suggest that most planets with masses greater than about $6 M_{\oplus}$ may contain a significant fraction of volatiles and/or $\mathrm{H} / \mathrm{He}$.

Kepler-454 has similar parameters to the Sun and does not appear to be unique compared to the other host stars in this sample. The transit parameters initially implied by CFOP placed Kepler-454b in a potentially unique space in the massradius diagram, with a radius estimate between the population of planets with Earth-like densities and the population of larger, less dense planets. With its radius measurement now revised upward due to analysis of the short cadence observation, Kepler-454b has both a mass and radius comparable to several of the less dense planets. At $2.76 \pm 0.73 \mathrm{~g} \mathrm{~cm}^{-3}$, it falls well above the Earth-like composition curve and it likely requires a significant fraction of volatiles.

Specifically, a planet with an Earth-like composition and the same mass as Kepler-454b would have a radius of $R_{\mathrm{p} \text {,Earth-like }}=1.73 R_{\oplus}$, significantly smaller than the observed radius of Kepler-454b $\left(R_{\mathrm{p}, \mathrm{obs}}=2.37 \pm 0.13 R_{\oplus}\right)$. The 

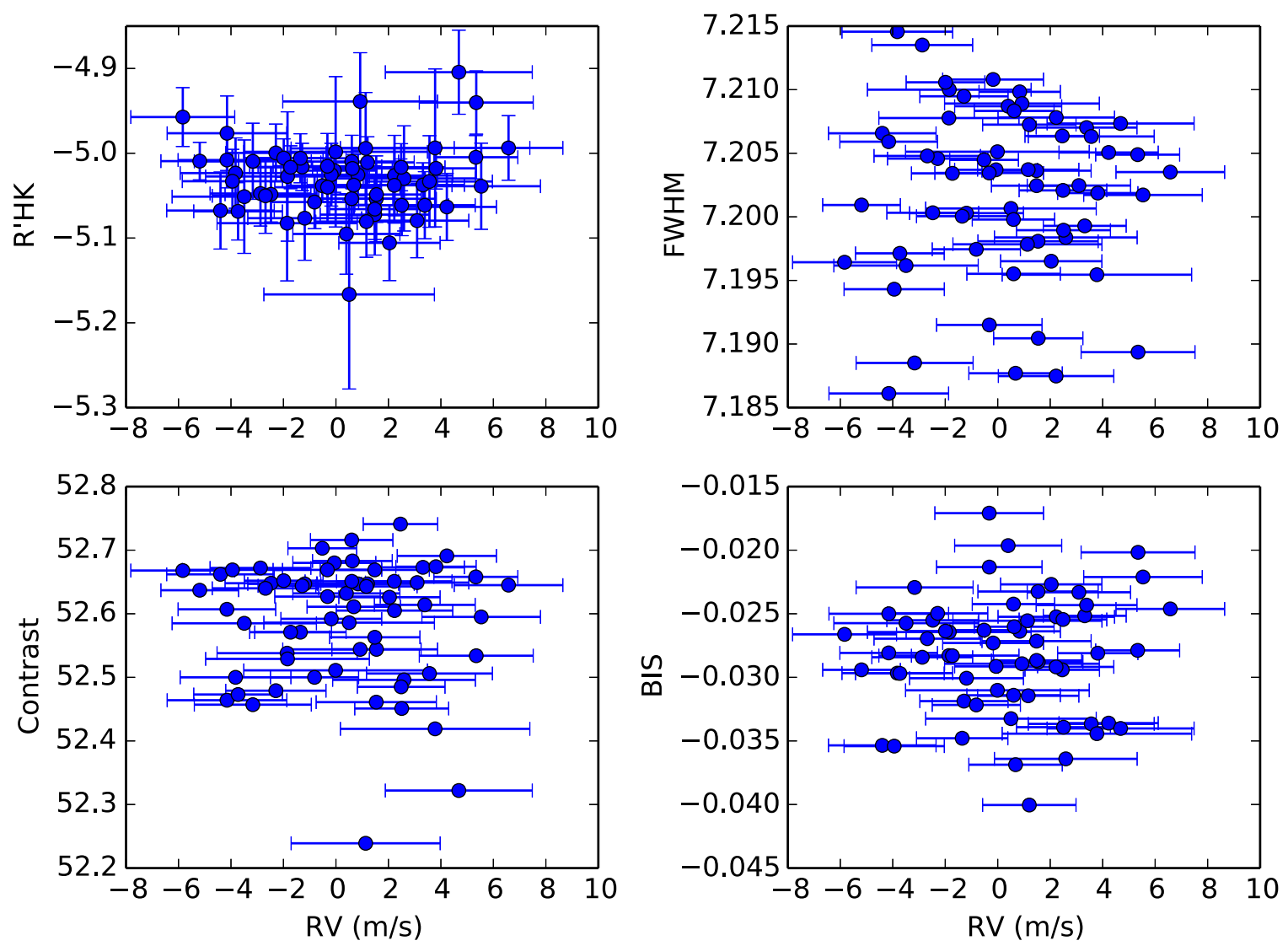

Figure 10. Plots of the $\log R_{\mathrm{HK}}^{\prime}$ activity index and the FWHM, contrast, and bisector velocity span of the cross-correlation function as a function of the de-trended radial velocity measurements. No correlations are found.

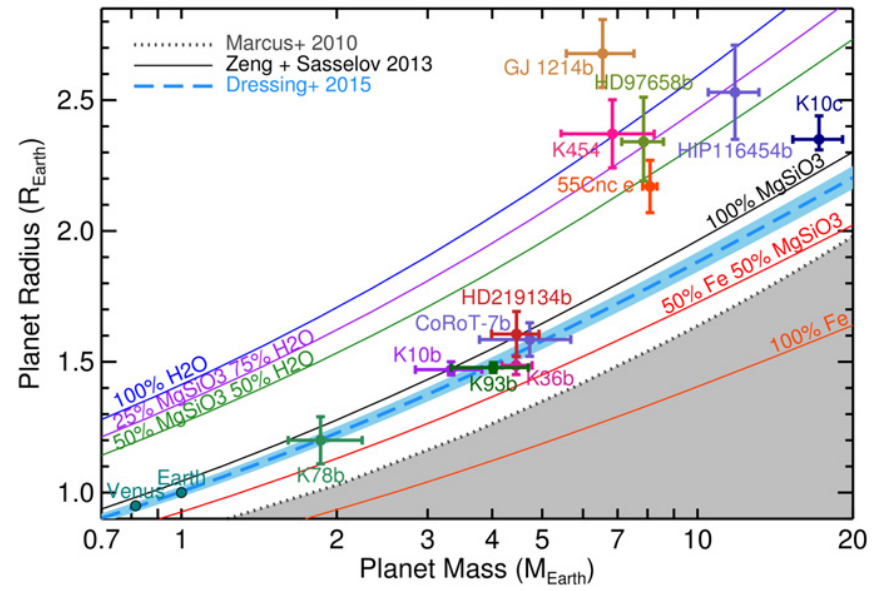

Figure 11. Mass-radius diagram for planets with radii $<2.7 R_{\oplus}$ and masses measured to better than $20 \%$ precision. The shaded gray region in the lower right indicates planets with iron content exceeding the maximum value predicted from models of collisional stripping (Marcus et al. 2010). The solid lines are theoretical mass-radius curves (Zeng \& Sasselov 2013) for planets with compositions of $100 \% \mathrm{H}_{2} \mathrm{O}$ (blue), $25 \% \mathrm{MgSiO}_{3}-75 \% \mathrm{H}_{2} \mathrm{O}$ (purple), $50 \% \mathrm{MgSiO}_{3}-50 \% \mathrm{H}_{2} \mathrm{O}$ (green), $100 \% \mathrm{MgSiO}_{3}$ (black), $50 \% \mathrm{Fe}-50 \%$ $\mathrm{MgSiO}_{3}$ (red), and $100 \% \mathrm{Fe}$ (orange). Our best-fit relation based on the Zeng \& Sasselov (2013) models is the dashed light blue line representing an Earth-like composition (modeled as $17 \% \mathrm{Fe}$ and $83 \% \mathrm{MgSiO}_{3}$ using a fully differentiated, two-component model). The shaded region surrounding the line indicates the $2 \%$ dispersion in the radius expected from the variation in $\mathrm{Mg} / \mathrm{Si}$ and $\mathrm{Fe} / \mathrm{Si}$ ratios (Grasset et al. 2009).

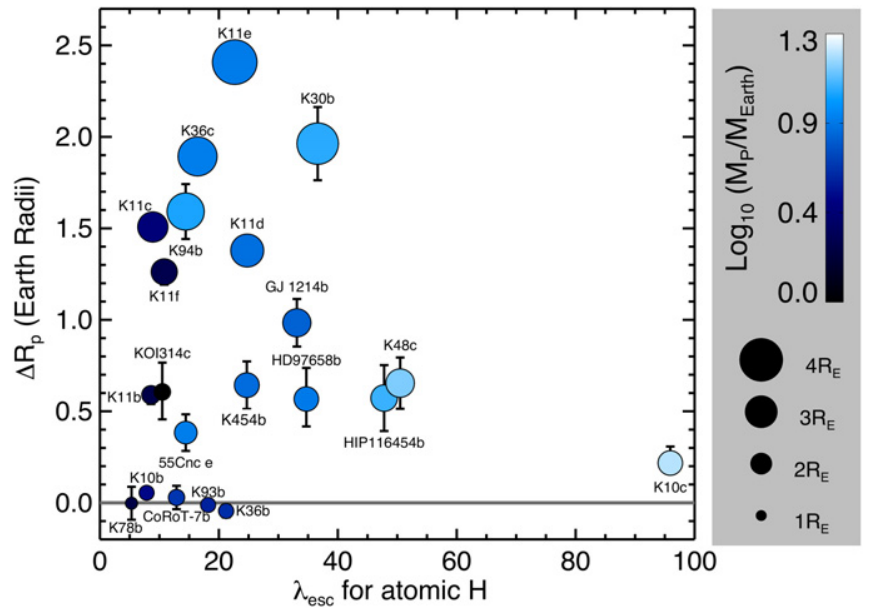

Figure 12. Difference between observed planet radius and the radius that would have been expected if the planet had an Earth-like composition vs. the Jeans escape parameter. Planets consistent with Earth-like compositions are concentrated near the gray line at $\Delta R_{\mathrm{p}}=0$. The sizes of the circles are scaled based on the radii of the planets and the colors indicate the planet masses.

observed "radius excess" $\Delta R_{\mathrm{p}}=R_{\mathrm{p}, \text { Earth-like }}-R_{\mathrm{p}, \mathrm{obs}}$ for Kepler- $454 \mathrm{~b}$ is therefore $\Delta R_{\mathrm{p}}=0.64 R_{\oplus}$.

We can estimate the amount of lower density material required to explain the observed radius of Kepler-454b by assuming that Kepler-454b is an Earth-like mixture of rock and 


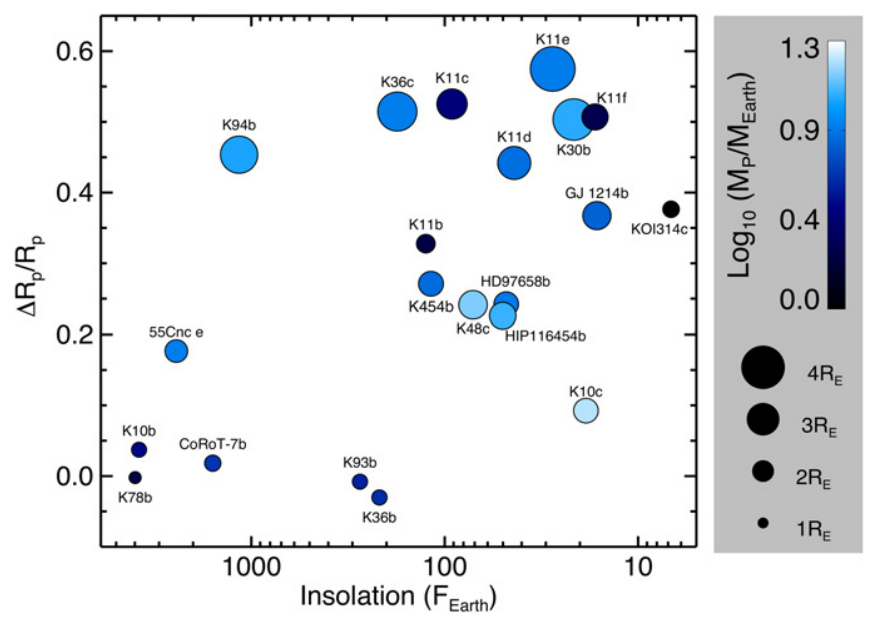

Figure 13. The relative radius excess $\left(\Delta R_{\mathrm{p}} / R_{\mathrm{p}}\right)$ vs. the insolation flux received by each planet. As in Figure 12, the sizes and colors of the circles indicate the radii and masses of the planets, respectively.

iron covered by a low density envelope. Employing the models of Lopez \& Fortney (2014) and assuming a system age of 5 Gyr, the observed mass and radius of Kepler- $454 \mathrm{~b}$ could be explained if the planet is shrouded by a solar metallicity $\mathrm{H} / \mathrm{He}$ and a total mass equal to roughly $1 \%$ of the total planetary mass.

In Figure 12, we compare the observed radius excess $\Delta R_{\mathrm{p}}=R_{\mathrm{p}, \text { obs }}-R_{\mathrm{p} \text {,Earth-like }}$ for Kepler- $454 \mathrm{~b}$ to the $\Delta R_{\mathrm{p}}$ estimated for other small transiting planets with mass estimates. The left panel of Figure 12 displays the $\Delta R_{\mathrm{p}}$ as a function of the Jeans escape parameter $\lambda_{\text {esc }}$ :

$$
\lambda_{\mathrm{esc}} \equiv \frac{G M_{\mathrm{p}} m}{k T r_{\mathrm{c}}},
$$

where $G$ is the gravitational constant, $M_{\mathrm{p}}$ is the mass of the planet, $m$ is the mean molecular or atomic weight of the atmosphere (here we set $m$ to the value of atomic hydrogen), $k$ is the Boltzmann constant, $r_{\mathrm{c}}$ is the height above the center of the planet, and $T$ is the temperature of the exobase, the atmospheric boundary above which particles are gravitationally bound to the planet but move on collision-free trajectories (Meadows \& Seager 2010). We assumed that the temperatures $T$ of the exobase are equal to the expected equilibrium temperatures of the planets for an albedo of 0 , but the true exobase temperatures are likely higher. For highly irradiated planets, the dominant atmospheric loss channel is likely hydrodynamic escape rather than Jeans escape. Accordingly, Figure 13 presents the ratio $\Delta R_{\mathrm{p}} / R_{\mathrm{p}}$ versus the insolation received by each planet. Compared to the other planets, Kepler454b is most similar to HD 97658b, HIP 116454b, Kepler 48c, and Kepler-11b. All of these planets receive roughly $100 \times$ the insolation received by the Earth and have relative radius excesses $\Delta R_{\mathrm{p}} / R_{\mathrm{p}}$ of approximately $20 \%$, consistent with prior work by Lopez et al. (2012) and Owen \& Jackson (2012).

For $\mathrm{H} / \mathrm{He}$ dominated atmospheric envelopes, $\Delta R_{\mathrm{p}} / R_{\mathrm{p}}$ is roughly equivalent to the relative mass fraction of envelope (Lopez \& Fortney 2014). In general, we find that the planets with lower relative envelope fractions are more highly irradiated than the planets with large relative envelope fractions. However, accurately constraining the masses and radii of small planets becomes increasingly difficult as the orbital period increases. The relative lack of small dense planets receiving low insolation fluxes may therefore be due to an observational bias rather than a real scarcity of cool dense small planets. As the precision of RV spectrographs improves, we may discover additional planets that are smaller, denser, and cooler than Kepler-10c. The NASA TESS Mission, scheduled for launch in 2017, will help by providing hundreds of Earths, super-Earths, and mini-Neptunes transiting stars that are generally much brighter than those from Kepler (Sullivan et al. 2015), greatly facilitating RV follow-up and permitting masses to be measured for much longer orbital periods.

In contrast, obtaining a precise mass measurement of a highly irradiated planet with a large relative envelope fraction is observationally easier than measuring the mass of a less strongly irradiated large planet. Accordingly, the relative dearth of highly irradiated small planets with large envelope fractions likely indicates that such planets are rare. As the number of small planets with well-measured masses and radii continues to grow, we will be able to further investigate the properties and the formation, and subsequently loss or retention of gaseous envelopes of small planets.

The authors would like to thank the TNG observers who contributed to the measurements reported here, including Walter Boschin, Massimo Cecconi, Vania Lorenzi and Marco Pedani. We also thank Lauren Weiss for gathering some of the HIRES data presented here. The authors wish to thank the entire Kepler team, without whom these results would not be possible. Funding for this Discovery mission is provided by NASA's Science Mission Directorate. The HARPS-N project was funded by the Prodex Program of the Swiss Space Office (SSO), the Harvard University Origin of Life Initiative (HUOLI), the Scottish Universities Physics Alliance (SUPA), the University of Geneva, the Smithsonian Astrophysical Observatory (SAO), and the Italian National Astrophysical Institute (INAF), University of St. Andrews, Queen's University Belfast and University of Edinburgh. The research leading to these results has received funding from the European Union Seventh Framework Programme (FP7/2007-2013) under grant Agreement No. 313014 (ETAEARTH). This publication was made possible by a grant from the John Templeton Foundation. The opinions expressed in this publication are those of the authors and do not necessarily reflect the views of the John Templeton Foundation. This material is based upon work supported by the National Aeronautics and Space Administration under grant No. NNX15AC90G issued through the Exoplanets Research Program. C.D. is supported by a National Science Foundation Graduate Research Fellowship. Work by C.D. was performed in part under contract with the California Institute of Technology (Caltech)/Jet Propulsion Laboratory (JPL) funded by NASA through the Sagan Fellowship Program executed by the NASA Exoplanet Science Institute. X.D. would like to thank the Swiss National Science Foundation (SNSF) for its support through an Early Postdoc Mobility fellowship. A.V. is supported by the National Science Foundation Graduate Research Fellowship, grant No. DGE 1144152. P. F. acknowledges support by Fundação para a Ciência e a Tecnologia (FCT) through Investigador FCT contracts of reference IF/ 01037/2013 and POPH/FSE (EC) by FEDER funding through the program "Programa Operacional de Factores de Competitividade-COMPETE". P.F. further acknowledges support 
from Fundação para a Ciência e a Tecnologia (FCT) in the form of an exploratory project of reference IF/01037/ 2013CP1191/CT0001. W.J.C., T.L.C. and G.R.D. acknowledge the support of the UK Science and Technology Facilities Council (STFC). S.B. acknowledges partial support from NSF grant AST-1105930 and NASA grant NNX13AE70G. T.S.M. was supported by NASA grant NNX13AE91G. Computational time on Stampede at the Texas Advanced Computing Center was provided through XSEDE allocation TG-AST090107. Funding for the Stellar Astrophysics Centre is provided by The Danish National Research Foundation (grant agreement No.: DNRF106). The research is supported by the ASTERISK project (ASTERoseismic Investigations with SONG and Kepler) funded by the European Research Council (grant agreement No.: 267864); and by the European Community's Seventh Framework Programme (FP7/2007-2013) under grant agreement No. 312844 (SPACEINN). Partial support was received from the Kepler mission under NASA Cooperation Agreement NNX13AB58A to the Smithsonian Astrophysical Observatory (PI:DWL).

Some of the data presented herein were obtained at the W. M. Keck Observatory, which is operated as a scientific partnership among the California Institute of Technology, the University of California, and the National Aeronautics and Space Administration. The Keck Observatory was made possible by the generous financial support of the W. M. Keck Foundation. The spectra and their products are made available at the NExSci Exoplanet Archive and its CFOP website: http://exoplanetarchive.ipac.caltech.edu. We thank the many observers who contributed to the HIRES measurements reported here, including Benjamin J. Fulton, Evan Sinukoff, and Lea Hirsch. We gratefully acknowledge the efforts and dedication of the Keck Observatory staff, especially Greg Doppmann, Scott Dahm, Hien Tran, and Grant Hill for support of HIRES and Greg Wirth and Bob Goodrich for support of remote observing. This research has made use of the NASA Exoplanet Archive, which is operated by the California Institute of Technology, under contract with the National Aeronautics and Space Administration under the Exoplanet Exploration Program. Finally, the authors wish to extend special thanks to those of Hawaiian ancestry on whose sacred mountain of Mauna Kea we are privileged to be guests. Without their generous hospitality, the Keck observations presented herein would not have been possible.

\section{REFERENCES}

Baranne, A., Queloz, D., Mayor, M., et al. 1996, A\&AS, 119, 373 Batalha, N. M., Rowe, J. F., Bryson, S. T., et al. 2013, ApJS, 204, 24 Bedding, T. R., \& Kjeldsen, H. 2010, CoAst, 161, 3

Bonomo, A. S., Sozzetti, A., Lovis, C., et al. 2014, A\&A, 572, A2 Borucki, W. J., Koch, D. G., Basri, G., et al. 2011, ApJ, 736, 19 Buchhave, L. A., Bizzarro, M., Latham, D. W., et al. 2014, Natur, 509, 593 Buchhave, L. A., Latham, D. W., Johansen, A., et al. 2012, Natur, 486, 375 Burke, C. J., Bryson, S. T., Mullally, F., et al. 2014, ApJS, 210, 19 Butler, R. P., Marcy, G. W., Williams, E., et al. 1996, PASP, 108, 500 Castelli, F., \& Kurucz, R. L. 2004, arXiv:astro-ph/0405087 Christensen-Dalsgaard, J. 2008, Ap\&SS, 316, 113

Cosentino, R., Lovis, C., Pepe, F., et al. 2012, Proc. SPIE, 8446, 1 Cutri, R. M., Skrutskie, M. F., van Dyk, S., et al. 2003, yCat, 2246, 0 Danby, J. M. A. 1988, Fundamentals of Celestial Mechanics (2nd ed.; Richmond, Va., USA: Willmann-Bell)

Davies, G. R., Bedding, T., Silva Aguirre, V., et al. 2015, MNRAS, in press (arXiv:1511.02105)
Davies, G. R., Handberg, R., Miglio, A., et al. 2014, MNRAS, 445, L94 Delfosse, X., Forveille, T., Ségransan, D., et al. 2000, A\&A, 364, 217

Demarque, P., Guenther, D. B., Li, L. H., Mazumdar, A., \& Straka, C. W. 2008, Ap\&SS, 316, 31

Dressing, C. D., \& Charbonneau, D. 2015, ApJ, 807, 45

Dressing, C. D., Charbonneau, D., Dumusque, X., et al. 2015, ApJ, 800,135

Dumusque, X., Bonomo, A. S., Haywood, R. D., et al. 2014, ApJ, 789, 154

Els, S., Lock, T., Comoretto, G., et al. 2014, Proc. SPIE, 9150, 0

Ford, E. B. 2005, AJ, 129, 1706

Ford, E. B. 2006, ApJ, 642, 505

Foreman-Mackey, D., Hogg, D. W., Lang, D., \& Goodman, J. 2013, PASP, 125,306

Fortney, J. J., Marley, M. S., \& Barnes, J. W. 2007, ApJ, 659, 1661

Fressin, F., Torres, G., Charbonneau, D., et al. 2013, ApJ, 766, 81

Furesz, G. 2008, PhD thesis, Univ. Szeged

Gelman, A., \& Rubin, D. B. 1992, StaSc, 7, 457

Gilks, W. R., Richardson, S., \& Spiegelhalter, D. J. 1995, Markov Chain Monte Carlo in Practice (Boca Raton: Chapman and Hall/CRC)

Gilliland, R. L., McCullough, P. R., Nelan, E. P., et al. 2011, ApJ, 726, 2

Grasset, O., Schneider, J., \& Sotin, C. 2009, ApJ, 693, 722

Handberg, R., \& Lund, M. N. 2014, MNRAS, 445, 2698

Henning, W. G., O’Connell, R. J., \& Sasselov, D. D. 2009, ApJ, 707, 1000

Høg, E., Fabricius, C., Makarov, V. V., et al. 2000, A\&A, 355, L27

Howard, A. W., Johnson, J. A., Marcy, G. W., et al. 2009, ApJ, 696, 75

Huber, D., Chaplin, W. J., Christensen-Dalsgaard, J., et al. 2013, ApJ, 767,127

Kass, R. E., \& Raftery, A. E. 1995, Journal of the American Statistical Association, 430, 773

Kipping, D. M. 2013, MNRAS, 435, 2152

Kipping, D. M. 2014, MNRAS, 440, 2164

Kovács, G., Zucker, S., \& Mazeh, T. 2002, A\&A, 391, 369

Kurucz, R. L. 1992, in IAU Symp., Vol. 149, The Stellar Populations of Galaxies, ed. B. Barbuy, \& A. Renzini (Dordrecht: Kluwer)

Léger, A., Selsis, F., Sotin, C., et al. 2004, Icar, 169, 499

Lopez, E. D., \& Fortney, J. J. 2014, ApJ, 792, 1

Lopez, E. D., Fortney, J. J., \& Miller, N. 2012, ApJ, 761, 59

Mamajek, E. E., \& Hillenbrand, L. A. 2008, ApJ, 687, 1264

Mandel, K., \& Agol, E. 2002, ApJL, 580, L171

Marcus, R. A., Sasselov, D., Hernquist, L., \& Stewart, S. T. 2010, ApJL, 712, L73

Marcy, G. W., Isaacson, H., Howard, A. W., et al. 2014, ApJS, 210, 20

Mayor, M., Pepe, F., Queloz, D., et al. 2003, Msngr, 114, 20

Mayor, M., \& Udry, S. 2008, PhST, 130, 014010

McQuillan, A., Mazeh, T., \& Aigrain, S. 2013, ApJL, 775, L11

Meadows, V., \& Seager, S. 2010, in Terrestrial Planet Atmospheres and Biosignatures, ed. S. Seager (Tuscon, AZ: Univ. of Arizona Press), 441

Metropolis, N., Rosenbluth, A. W., Rosenbluth, M. N., Teller, A. H., \& Teller, E. 1953, JChPh, 21, 1087

Morton, T. D., \& Johnson, J. A. 2011, ApJ, 738, 170

Motalebi, F., Udry, S., Gillon, M., et al. 2015, arXiv:1507.08532

Owen, J. E., \& Jackson, A. P. 2012, MNRAS, 425, 2931

Pepe, F., Mayor, M., Rupprecht, G., et al. 2002, Msngr, 110, 9

Rowe, J. F., Coughlin, J. L., Antoci, V., et al. 2015, ApJS, 217, 16

Seager, S., Kuchner, M., Hier-Majumder, C. A., \& Militzer, B. 2007, ApJ, 669, 1279

Silva Aguirre, V., Davies, G. R., Basu, S., et al. 2015, MNRAS, 452, 2127

Sliski, D. H., \& Kipping, D. M. 2014, ApJ, 788, 148

Sneden, C. 1973, ApJ, 184, 839

Sousa, S. G., Santos, N. C., Adibekyan, V. Z., Delgado Mena, E., \& Israelian, G. 2015, A\&A, 577, A67

Sousa, S. G., Santos, N. C., Israelian, G., Mayor, M., \& Udry, S. 2011, A\&A, 533,141

Sullivan, P. W., Winn, J. N., Berta-Thompson, Z. K., et al. 2015, ApJ, 809, 77

Tull, R. G., MacQueen, P. J., Sneden, C., \& Lambert, D. L. 1995, PASP, 107,251

Tuomi, M., \& Jones, H. R. A. 2012, A\&A, 544, A116

Valencia, D., O'Connell, R. J., \& Sasselov, D. 2006, Icar, 181, 545

Vogt, S. S., Allen, S. L., Bigelow, B. C., et al. 1994, Proc. SPIE, 2198, 362

Weiss, A., \& Schlattl, H. 2008, Ap\&SS, 316, 99

White, T. R., Bedding, T. R., Gruberbauer, M., et al. 2012, ApJL, 751, L36

White, T. R., Bedding, T. R., Stello, D., et al. 2011, ApJ, 743, 161

Zeng, L., \& Sasselov, D. 2013, PASP, 125, 227

Zhang, K., \& Hamilton, D. P. 2008, Icar, 193, 267 